UvA-WINS-Wisk-99-10

LMU-TPW 99-13

hep-th/9907112

\title{
Anomaly cancellation in K3 orientifolds
}

\author{
Claudio A. Scrucca ${ }^{a, 1}$ And Marco Serone ${ }^{b, c, 2}$ \\ a Department of Physics, Ludwig Maximilian University of Munich \\ Theresienstraße 37, 80333 Munich, Germany \\ ${ }^{b}$ Department of Mathematics, University of Amsterdam \\ Plantage Muidergracht 24, 1018 TV Amsterdam, The Netherlands \\ ${ }^{c}$ Spinoza Institute, University of Utrecht \\ Leuvenlaan 4, 3584 CE Utrecht, The Netherlands
}

\begin{abstract}
We study in detail the pattern of anomaly cancellation in $D=6$ Type IIB $\mathbf{Z}_{N}$ orientifolds, occurring through a generalized Green-Schwarz mechanism involving several RR antisymmetric tensors and scalars fields. The starting point is a direct string theory computation of the inflow of anomaly arising from magnetic interaction of D-branes, O-planes and fixed-points, which are encoded in topological one-loop partition functions in the RR odd spin-structure. All the RR anomalous couplings of these objects are then obtained by factorization. They are responsible for a spontaneous breaking of $U(1)$ factors through a Higgs mechanism involving the corresponding hypermultiplets. Some of them are also related by supersymmetry to gauge couplings involving the NSNS scalars sitting in the tensor multiplets. We also comment on the possible occurrence of tensionless strings when these couplings diverge.
\end{abstract}

\footnotetext{
${ }^{1}$ Claudio.Scrucca@physik. uni-muenchen.de

2 serone@wins.uva.nl
} 


\section{Introduction}

Anomalies have proven to play a prominent role in the study of non-trivial vacua of string theory. Their cancellation constitute a very severe constraint on the lowenergy quantum field theory, which can be usually understood in string theory as consequence of basic consistency requirements, like modular invariance and tadpole cancellation. A very interesting peculiarity of low-energy effective quantum field theories emerging from string theory is the occurrence of anomalies in certain tree-level magnetic interaction mediated by p-form gauge fields, beside the well known anomalies arising in one-loop amplitudes involving chiral fermions or self-dual bosons. This allows for consistent quantum fields theories with a non-vanishing one-loop anomaly cancelled by an equal and opposite tree-level anomaly. This is of course the celebrated Green-Schwarz mechanism [1].

Thanks to the important recent developments in string theory, this cancellation mechanism can be understood as a particular example of the so-called inflow mechanism [2]. Given a consistent anomaly free theory, there can exist vacua with topological defects supporting anomalous zero modes. The latter will be responsible for a local violation of charge conservation on the defects, which has to be compensated by an inflow of charge from outside the defects induced by appropriate couplings of the defects to the fields living in the bulk. In string theory, this kind of non-perturbative objects do indeed arise in non-trivial vacua, important examples being D-branes and O-planes [3]. Both of these kind of objects support anomalous fields on their worldvolumes, and have thus to have appropriate anomalous couplings to bulk fields for consistency. Indeed, they present topological Wess-Zumino couplings to RR p-forms which are precisely those required to cancel through the inflow mechanism all the one-loop anomalies on their world-volumes [ [, 5, 6]. The effective appearance of all of these couplings in string theory has been deduced in a unified way in [7] by factorizing RR magnetic interactions between D-branes and O-planes with non trivial curvatures, encoded in annulus, Möbius strip and Klein bottle amplitudes. They have also been checked in successive steps through direct computations of the induced RR charges, encoded in disk and crosscap amplitudes [8, 9, 10, 11]. Some terms have also been derived through duality [12, 13, 14]. In orientifold models [15, 16, 17, 18], the situation is particularly clear. The D-branes associated to Chan-Paton degrees of freedom, as well as the fixed-planes of the orientifold group action like O-planes, support in general anomalous fields giving rise to a non-vanishing anomaly, but also appropriate Wess-Zumino couplings to RR fields inducing a net inflow of anomaly. For consistent models, these one-loop and tree-level anomalies always cancel; this is usually a direct consequence of the more basic consistency requirement of tadpole cancellation.

In this paper, we study in detail anomaly cancellation in Type IIB $\mathbf{Z}_{N}$ orientifolds in $D=6$ dimensions [16, 19, 20, 21, 22]. These models are particularly interesting 
because of their rich and peculiar spectrum, which differs from what expected [23] for a smooth Type IIB orientifold on $K 3$, which is equivalent to Type I on $K 3$ as a consequence of the well known fact that Type I strings can be understood as the simplest orientifold of Type IIB strings [15]. In particular, a variable number of extra tensor multiplets arise in addition to the universal one expected in the smooth case [19]. Anomaly cancellation imposes extremely powerful constraints on these theories, like on every $N=(1,0)$ supersymmetric chiral theory in $D=6$, due to the potential presence of both gauge and gravitational anomalies [24. In general, a generalization of the Green-Schwarz mechanism involving all the RR fields present in the model occurs [25]. In [6], a systematic way of studying anomaly cancellation in orientifold models was sketched, and applied to the simple case of the $\mathbf{Z}_{2}$ model, investigated in great detail in [26], in which no extra tensor multiplets arise]. This work is devoted to a detailed analysis of all the $\mathbf{Z}_{N}$ models, with particular emphasis on the inflow interpretation, for the case of maximal unbroken gauge group. We will proceed with a refinement of the philosophy used in [0, 6], consisting in a direct string computation of the inflow of anomaly arising from magnetic interaction among D-branes, O-planes and fixed-points. All the anomalous couplings in each model can then be easily obtained by factorization. Due to supersymmetry, these give an important information on other terms of the low-energy effective action, in particular to the form of the gauge couplings $1 / g^{2}$ present in the theory [25]. Interestingly, there are special points in the moduli space of the tensor multiplet scalars where $1 / g^{2}$ vanishes or becomes negative [25]; tensionless strings appear then in the spectrum and the theory may undergo a phase transition [28, 29, 30]. The orientifold models discussed here correspond to particular points in the moduli space parametrized by these scalars, where all the gauge couplings are positive definite and finite. However, from some coefficients appearing in the anomaly polynomial and by supersymmetry, one can find the dependence of the gauge couplings on these moduli. We find points where these are vanishing and no simple string description is available. One can also fix the couplings between these scalars and the gauge fields. The results are in agreement with what found in [31], where such couplings were computed for the D9-brane gauge group by factorizing one-loop CP-even amplitudest 2 .

Analogously to what has been shown for the $\mathbf{Z}_{2}$ orientifold in [26], anomaly cancellation involves also the exchange of scalar fields and their dual four-forms. This results in the breaking of some (but in general not all, even in the case of maximal gauge symmetry discussed here) of the $U(1)$ gauge factors present in the gauge group.

This paper is organized as follows. In section two, we explain the strategy used to compute the inflow of anomaly through one-loop computations in the RR odd spin-structure. In section three, we apply the general results obtained in section two

\footnotetext{
${ }^{1}$ Anomaly cancellation in $D=4 N=1$ orientifolds has been studied in [27].

${ }^{2}$ See again [27] for similar couplings in $D=4 N=1$ models.
} 
to the $\mathbf{Z}_{N}$ orientifolds. In section four, we find the anomalous couplings of D-branes, O-planes and fixed-points by factorization, and in section five we study explicitly the factorization of the anomaly for each model. In section six, we analyze the form of some terms in the low-energy effective action of these models, related by supersymmetry to the anomalous couplings found previously, and the spontaneous breaking of some $U(1)$ factors of the gauge group. Finally, in last section, we give some conclusions and in the two appendices we respectively recall useful formulae about the inflow mechanism and report the explicit form of the anomalous couplings for each model.

\section{Inflow and anomalies in string theory}

One-loop anomalies in quantum field theory as well as in string theory can be computed by evaluating one-loop diagrams with suitable external particles (gravitons and gauge fields for the case of gravitational and gauge anomalies), one of which polarized longitudinally. Consistent string theories are known to be anomaly-free. At the level of their low-energy effective actions, the total one-loop anomaly need not to vanish, but can cancel against anomalies due to particular couplings between gauge particles and RR fields. The crucial feature of these actions is that these couplings do actually appear and precisely in the appropriate form to give an anomaly free quantum field theory. One could be satisfied by this statement and conclude that such couplings can be derived by requiring the tree-level anomaly they produce to be equal and opposite to the one-loop anomaly produced by the chiral fields present in the model. Such point of view is however unsatisfactory for at least two reasons. First, it is indirect and always relies on the assumption (generically taken to be valid) that consistent string theories are anomaly free. Most importantly, it does not allow for a microscopic analysis of the origin of such couplings in string theory. This second reason is crucial for our purposes of computing anomalous couplings in IIB orientifolds. It is expected, for instance, that in such models several tensor and hyper multiplets, coming from different twisted closed string sectors, play a decisive role in the inflow mechanism. Whereas a string derivation of these couplings allows a total understanding of their origin and the role they play in the various inflows, an analysis at the level of the low-energy effective action only, would be necessarily incomplete.

The question is then how to compute such anomalous couplings (or the corresponding inflows) in string theory, and more precisely in generic Type IIB orientifolds. The most direct approach would be to perform a disk or crosscap computation with the insertion of gauge fields, gravitons and the RR field in question. The advantage of this approach is that it yields directly the couplings. However, the precise form of the vertex operators of the various tensor fields is needed, including those arising from the closed string twisted sectors, and it is quite awkward and hard to fix correctly the normalizations. On the other hand, one can compute appropriate amplitudes from 
which such couplings can be extracted by factorization. The most convenient choice turns out to be a generic one-loop correlation function in the odd spin-structure with external gravitons and gauge fields, one of which polarized longitudinally. These amplitudes on the annulus, Möbius strip and Klein bottle can indeed be factorized as a tree-level exchange of closed strings between two disk or crosscap sources. As we shall argue in the following, this amounts to compute directly the anomalous inflows induced by these couplings.

Before entering into the details of the computation, we would like to do some remarks and anticipate some results. The correlation functions above will turn out to be a total derivative in the moduli space of the various relevant surfaces: annulus, Möbius strip and Klein bottle. As well known, these surfaces have two equivalent interpretations, either as one-loop amplitudes of open (for the annulus and Möbius strip) or closed (for the Klein bottle) strings, or as tree-level closed string amplitudes. Correspondingly, the modular parameter of these surfaces can be taken either as the proper time $t$ in the loop channel or the proper time $l \sim 1 / t$ in the tree channel. The inflow due to massless RR fields will arise from the IR region $l \rightarrow \infty$ of the treechannel, corresponding to the UV region $t \rightarrow 0$ of the loop channel, where indeed usual anomalies can arise.

Due to the topological nature of the amplitudes in question, the computation always reduces to the simple evaluation of the partition function of a supersymmetric quantum mechanical model. From now on, we will concentrate for simplicity on the case of six non-compact dimensions, since this is the case we are interested in. More general cases can be treated similarly without additional difficulties, the fields along the compact directions entering always through their odd spin-structure partition function. We are then interested to compute a given four-point function of gluons and/or gravitons in the odd spin-structure, with one of them polarized longitudinally. The unphysical particle represents essentially a gauge transformation, and choosing it to be a gluon or a graviton corresponds to compute gauge and gravitational anomalous variations respectively.

In the odd spin-structure, the genus one world-sheets we are considering admit a gravitino zero mode. Independently of the boundary conditions, the integration over the latter in the Polyakov path-integral results in the insertion of the sum of the left and right moving world-sheet supercurrents, $T_{F}+\tilde{T}_{F}$. Moreover, since the total superghost charge is 1 , one has to take one vertex operator in the $(-1)$-picture and all the others in the (0)-picture. A non-vanishing result is obtained by taking the vertex of the unphysical particle to be in the $(-1)$-picture and all the vertices of the physical particles in the (0)-picture. We have then to compute

$$
C=\left\langle V_{1}^{\text {phy }} V_{2}^{\text {phy. }} V_{3}^{\text {phy. }} V^{\text {unphy. }}\left(T_{F}+\tilde{T}_{F}\right)\right\rangle
$$

In the following, we will use the same strategy as in the introduction of [32]. For simplicity, we will systematically omit the ghost and superghost dependence of all 
the operators, since their contributions to the correlation functions we will compute always cancel by supersymmetry.

The vertex operators in the (0)-picture for physical gluons and gravitons, with transverse polarizations $\epsilon_{\mu}^{a}$ and $\xi_{\mu \nu}$, are the usual ones. They are given by

$$
\begin{aligned}
V_{\gamma}^{p h y} & =\xi_{\mu}^{a} \lambda^{a} \oint d \tau\left(\dot{X}^{\mu}+i p \cdot \psi \psi^{\mu}\right) e^{i p \cdot X} \\
V_{g}^{p h y} & =\xi_{\mu \nu} \int d^{2} z\left(\partial X^{\mu}+i p \cdot \psi \psi^{\mu}\right)\left(\bar{\partial} X^{\nu}+i p \cdot \tilde{\psi} \tilde{\psi}^{\nu}\right) e^{i p \cdot X}
\end{aligned}
$$

The vertex operators in the $(-1)$-picture for unphysical gluons and gravitons, with longitudinal polarizations $\epsilon_{\mu}^{a}=p_{\mu} \eta^{a}$ and $\xi_{\mu \nu}=p_{(\mu} \eta_{\nu)}$, are not total derivatives as they would be in the (0)-picture. However, they can be written as the world-sheet supersymmetry variations

$$
V^{u n p h y}=\left[Q+\tilde{Q}, \hat{V}^{u n p h y \cdot}\right]
$$

of the auxiliary vertices

$$
\begin{aligned}
& \hat{V}_{\gamma}^{\text {unphy. }}=-i \eta^{a} \lambda^{a} \oint d \tau e^{i p \cdot X} \\
& \hat{V}_{g}^{\text {unphy. }}=-2 i \eta_{\mu} \int d^{2} z\left[(\partial+\bar{\partial}) X^{\mu}+i p \cdot(\psi-\tilde{\psi})(\psi-\tilde{\psi})^{\mu}\right] e^{i p \cdot X} .
\end{aligned}
$$

In the above expressions, $\lambda^{a}$ are the Chan-Paton matrices, and

$$
Q=\oint_{C_{\tau}} \frac{d z}{2 \pi i} j, \quad \tilde{Q}=\oint_{C_{\tau}} \frac{d \bar{z}}{2 \pi i} \tilde{j},
$$

are the left and right-moving world-sheet supercharges.

We can now use standard manipulations as in 33 and reverse the contour integration for $Q$ and $\tilde{Q}[$. Since all the other vertex operators in the correlation are supersymmetric, the only non-vanishing term arises when $Q+\tilde{Q}$ hits the picture changing vertex operator $T_{F}+\tilde{T}_{F}$, giving

$$
\left[Q+\tilde{Q}, T_{F}+\tilde{T}_{F}\right]=T_{B}+\tilde{T}_{B}
$$

where $T_{B}+\tilde{T}_{B}$ is the world-sheet energy-momentum tensor. We are then left with

$$
C=\left\langle V_{1}^{p h y \cdot} V_{2}^{p h y \cdot} V_{3}^{p h y \cdot} \hat{V}^{u n p h y \cdot}\left(T_{B}+\tilde{T}_{B}\right)\right\rangle
$$

The insertion of the energy-momentum tensor in a correlation function corresponds generically to take a Teichmüller deformation of the remaining amplitude; in our case, this means basically to take a derivative with respect to the modulus $t$. By a careful treatment of the path-integral measure, it has been shown that this is indeed precisely what happens for the six-gluon correlation function giving the hexagon gauge anomaly in type I string theory 34]. It is not difficult to convince oneself that

\footnotetext{
${ }^{3}$ In the case at hand, these manipulations have to be taken on the covering torus of each surface.
} 
the same analysis can be carried out in the present case without any major change, the compact space entering rather trivially in (2.8). The final result for the integrated amplitude is then

$$
A=\int_{0}^{\infty} d t \frac{d}{d t}\left\langle V_{1}^{p h y \cdot} V_{2}^{\text {phy. }} V_{3}^{\text {phy. }} \hat{V}^{\text {unphy. }}\right\rangle
$$

This is indeed in agreement with the expectation that space-time anomalies in string theory arise as boundary terms in the moduli space of the various involved surfaces [33]. This limit involves also the position of the graviton vertices. A potential anomaly can arise only when all gravitons approach the same world-sheet boundary where all the gauge fields sit or, for pure gravitational anomalies, when the four gravitons are on the same boundary or crosscap [35]. These are the only kinematical configurations in which, along the whole one-loop surface, there is no flow of momentum $q$ in the tree-level channel leading to an exponential suppression of the amplitude like $e^{-q^{2} / t}$. This is nothing but a generalization of the argument used to show that non-planar diagrams, in the hexagon gauge anomaly of type I, are anomaly-free (see e.g. [36]). The boundary value we are interested in is $t \rightarrow 0$, that is $l \rightarrow \infty$, where the amplitude is dominated by the exchange of massless closed string states. The limit $t \rightarrow \infty$ corresponds instead to the IR part of the loop channel.

The full evaluation of (2.9), in particular when more gravitons are present, requires still a considerable amount of work. However, it is not our aim to perform such a calculation, that in a consistent string model has to give necessarily a vanishing resultf. Rather, we are interested to extract from (2.9) the various anomalous inflows coming from the exchange of RR fields. In order to do that, we can restrict the evaluation of (2.9) to the lowest order in the external momenta $p_{\mu}$. Indeed, in quantum field theory, any momentum dependence in an anomalous variation of the effective action can be removed by adding suitable local counter terms? This is not possible in string theory, where the full anomalous variation presents indeed a dependence on the external momenta, dependence that turns out to be crucial to get a total vanishing result. By neglecting this dependence we will then be able to extract a finite and non-vanishing result corresponding to the inflow terms. Notice that in this approximation the amplitude (2.9) is t-independent.

Recall now that the odd spin-structure one-loop correlation function above involves an integration over the six bosonic zero modes $x_{0}^{\mu}$, corresponding to the integral over six-dimensional space-time, as well as an integration over the six fermionic zero modes $\psi_{0}^{\mu}$, which is vanishing unless all of them are inserted. Notice that none of the unphysical vertices (2.5) and (2.6) contains fermionic zero modes, so that all of them must be provided by the physical vertices $V^{\text {phy }}$. As discussed in [7], the gluon

\footnotetext{
${ }^{4}$ See for example [35], where the mixed and gravitational anomalies are computed in type I string theory, using the covariant operatorial formalism, and are shown to vanish.

${ }^{5}$ Corrections involving higher powers of $p_{\mu}$ would be in any case sub-leading in the low-energy limit $\alpha^{\prime} \rightarrow 0$.
} 
and graviton vertex operators can soak at most two fermionic zero modes, and their effective form at leading order in the momentum $p^{\mu}$ is

$$
\begin{aligned}
& V_{\gamma}^{e f f \cdot(F)}=\oint d \tau F^{a} \lambda^{a}, \\
& V_{g}^{e f f \cdot(R)}=\int d^{2} z R_{\mu \nu}\left[X^{\mu}(\partial+\bar{\partial}) X^{\mu}+(\psi-\tilde{\psi})^{\mu}(\psi-\tilde{\psi})^{\nu}\right],
\end{aligned}
$$

in terms of the gauge and gravitational curvature two-forms

$$
F^{a}=\frac{1}{2} F_{\mu \nu}^{a} \psi_{0}^{\mu} \psi_{0}^{\nu}, \quad R_{\mu \nu}=\frac{1}{2} R_{\mu \nu \rho \sigma} \psi_{0}^{\rho} \psi_{0}^{\sigma}
$$

Interestingly enough, the unphysical gluon and graviton vertex operators reduce, at leading order in the momentum $p^{\mu}$, to effective vertices of exactly the same kind, but with unphysical longitudinal curvatures $F^{a}=\eta^{a}$ and $R_{\mu \nu}=p_{[\mu} \eta_{\nu]}$ which do not depend on the fermionic zero modes.

Since all the effective vertices are now at most quadratic in the fields, it is convenient to exponentiate them adding the corresponding interaction to the free worldsheet action. The net effect of the unphysical vertex is to shift the corresponding curvature as $F^{a} \rightarrow F^{a}+\eta^{a}$ if it is a gluon, or $R_{\mu \nu} \rightarrow R_{\mu \nu}+\mathcal{D}_{[\mu} \eta_{\nu]}$ if it is a graviton. The integral over the six fermionic zero modes automatically selects the correct number (three) of physical vertices or, in language of forms, the six-form polynomial to be integrated over the six-dimensional space-time we are considering. However, in order to get a single unphysical vertex, one has to take the functional derivative with respect to the parameter $\eta^{a}$ or $\eta^{\mu}$, and then set the parameter to zero. A striking similitude then emerges with Fujikawa's method of computing anomalies, as in [24]. The unphysical vertex plays the role of the anomalous variation of the path-integral measure, and implement essentially the descent operation $I=d I^{(0)}, \delta I^{(0)}=d I^{(1)}$, on an auxiliary eight-form anomaly polynomial $I$, as required by the Wess-Zumino consistency condition.

Interestingly, one can at this point forget about the descent parameter $\eta$ associated to the unphysical vertex, keeping in mind that its net effect is to implement the descent procedure, and compute directly the anomaly polynomial $I$ rather than the anomaly $A$ itself. To achieve this out of the remaining correlation, one restricts by hand to the eight-form instead of integrating over the six fermionic zero modes which would select the six-form, and omits also the integral over the six bosonic zero modes. We have therefore reduced the computation of the anomaly to the evaluation of a supersymmetric partition function in the odd spin-structure in presence of an arbitrary gravitational and gauge background. World-sheet supersymmetry further implies that this is an index [37] which is independent of the modulus $t$, receiving contribution only from zero energy states of the two-dimensional $\sigma$-model. The computation, as anticipated, reduces then to the evaluation of the partition function of a supersymmetric quantum mechanical model. The compact space plays a central role in determining the precise normalization factors of the polynomial; moreover, in the 
cases we will consider in which it is realized as an orbifold, it will induce additionally twists in the Chan-Paton degrees of freedom [20].

The most important outcome of the above reasoning is a simple and general prescription for the direct string theory computation of the anomaly inflow in arbitrary models, and in particular Type IIB orientifolds. By factorizing the annulus, Möbius strip and Klein bottle inflows, one can then deduce the anomalous couplings of all the D-branes, O-planes and fixed-points in the model. In general, such couplings involve several RR fields arising in all the twisted sectors, and a generalized inflow mechanism takes places. Correspondingly, the anomaly polynomial does not factorize, but rather splits into a sum of factorized contributions corresponding to a plethora of sub-inflows.

\section{Inflow for K3 orientifolds}

In this section, we shall apply the tools derived in the previous section to the $D=6$ Type IIB $\mathbf{Z}_{N}$ orientifolds of [20, 21, 22]. In these models, tadpole cancellation requires in general both D9-branes and D5-branes, and fixes their number. In the following, we shall restrict to the special points in the moduli space in which the models have maximal gauge symmetry. This implies in particular that all the D5branes sit at the fixed-point at the origin of the orbifold. For all the details about these models, we refer to 21].

In this paper, we will focus on the type $A \mathbf{Z}_{N}$ orientifolds, in the terminology of [21]. The $D=6$ spectrum consist of gravitational, tensor, hyper and vector multiplets of $N=(1,0)$ supersymmetry. The untwisted closed string sector yields the minimal combination of 1 gravitational, 1 tensor and 2 hyper multiplets for all $N \neq 2$. For $N=2$ we get 1 gravitational, 1 tensor and 4 hyper multiplets. The twisted closed string sector gives a varying number of neutral hyper multiplets and additional tensor multiplets. These closed string spectra are summarized below.

\begin{tabular}{|c|c|c|}
\hline Model & Neutral Hypers & Tensors \\
\hline $\mathbf{Z}_{2}$ & 20 & 1 \\
\hline $\mathbf{Z}_{3}$ & 11 & 10 \\
\hline $\mathbf{Z}_{4}$ & 16 & 5 \\
\hline $\mathbf{Z}_{6}$ & 14 & 7 \\
\hline
\end{tabular}

As a consequence of the fact that the total number of neutral hyper and tensor multiplets is always 21, there are just enough neutral anti-chiral spinors to combine with the chiral gravitino to give the same gravitational anomaly as 8 self-dual tensors, 
as follows from the six-dimensional anomaly cancellation relation

$$
I_{3 / 2}-21 I_{1 / 2}-8 I_{A}=0 .
$$

As a result, the total anomaly from neutral closed string states is

$$
I_{n}=\left(9-n_{T}\right) I_{A},
$$

where $n_{T}$ is the number of tensor multiplets in the model. This anomaly is expected to be canceled by the inflow associated to the fixed-plane-fixed-plane interaction encoded in the Klein bottle amplitude.

In the open string sectors, one has vector multiplets as well as charged hyper multiplets in various antisymmetric or bi-fundamental representations, as shown in

\begin{tabular}{|c|c|c|c|}
\hline Model & & Gauge Group & Charged Hypermultiplets \\
\hline $\mathbf{Z}_{2}$ & $\begin{array}{l}99 \\
55\end{array}$ & $\begin{array}{l}U(16) \\
U(16)\end{array}$ & $\begin{array}{ll}99: & 2 \times \mathbf{1 2 0} \\
55: & 2 \times \mathbf{1 2 0} \\
95: & (\mathbf{1 6}, \mathbf{1 6})\end{array}$ \\
\hline $\mathbf{Z}_{3}$ & 99 & $U(8) \times S O(16)$ & $99: \quad(\mathbf{2 8}, \mathbf{1}),(\mathbf{8}, \mathbf{1 6})$ \\
\hline $\mathbf{Z}_{4}$ & $\begin{array}{l}99 \\
55\end{array}$ & $\begin{array}{l}U(8) \times U(8) \\
U(8) \times U(8)\end{array}$ & $\begin{array}{ll}99: & (\mathbf{2 8}, \mathbf{1}),(\mathbf{1}, \mathbf{2 8}),(\mathbf{8}, \mathbf{8}) \\
55: & (\mathbf{2 8}, \mathbf{1}),(\mathbf{1}, \mathbf{2 8}),(\mathbf{8}, \mathbf{8}) \\
95: & (\mathbf{8}, \mathbf{1} ; \mathbf{8}, \mathbf{1}),(\mathbf{1}, \mathbf{8} ; \mathbf{1}, \mathbf{8})\end{array}$ \\
\hline $\mathbf{Z}_{6}$ & 99 & $\begin{array}{l}U(4) \times U(4) \times U(8) \\
U(4) \times U(4) \times U(8)\end{array}$ & $\begin{array}{ll}99: \quad(6, \mathbf{1}, \mathbf{1}),(\mathbf{1}, \mathbf{6}, \mathbf{1}) \\
& (\mathbf{4}, \mathbf{1}, \mathbf{8}),(\mathbf{1}, \mathbf{4}, \mathbf{8}) \\
55: \quad & (\mathbf{6}, \mathbf{1}, \mathbf{1}),(\mathbf{1}, \mathbf{6}, \mathbf{1}) \\
& (\mathbf{4}, \mathbf{1}, \mathbf{8}),(\mathbf{1}, \mathbf{4}, \mathbf{8}) \\
95: \quad & (\mathbf{4}, \mathbf{1}, \mathbf{1} ; \mathbf{4}, \mathbf{1}, \mathbf{1}) \\
& (\mathbf{1}, \mathbf{4}, \mathbf{1} ; \mathbf{1}, \mathbf{4}, \mathbf{1}) \\
& (\mathbf{1}, \mathbf{1}, \mathbf{8} ; \mathbf{1}, \mathbf{1}, \mathbf{8})\end{array}$ \\
\hline
\end{tabular}
the following table.

In computing the anomalies produced by these fields, it is convenient to decompose all the representations as tensor products of two fundamental representations (associated to the end-points of open strings). Correspondingly, the Chern classes appearing in the anomaly decompose as products of traces in the fundamental representation. We shall indicate the latter with $c(F)$ in the following; more precisely, for the groups $U(n)$ and $S O(n)$ that appear we define

$$
c(F)=\operatorname{ch}_{\mathbf{n}}(F)=\operatorname{tr}_{\mathbf{n}}\left[e^{i F / 2 \pi}\right] .
$$


For $U(n)$, the adjoint, symmetric and antisymmetric representations give

$$
U(n):\left\{\begin{array}{l}
\operatorname{ch}_{\mathbf{n}^{2}}(F)=c(F) c(-F) \\
\operatorname{ch}_{\frac{\mathbf{n}(\mathbf{n} \pm \mathbf{1}}{2}}(F)=\frac{1}{2}\left[c(F)^{2} \pm c(2 F)\right]
\end{array} .\right.
$$

For $S O(n)$, the adjoint and symmetric representations give similarly

$$
S O(n): \operatorname{ch}_{\frac{\mathbf{n}(\mathbf{n} \pm 1)}{2}}(F)=\frac{1}{2}\left[c(F)^{2} \pm c(2 F)\right] .
$$

Using these relations, the total gauge and gravitational anomalies produced by the charged open string states in the various sectors can be easily computed. The anomaly

\begin{tabular}{|c|c|}
\hline Model & $I_{c}$ \\
\hline $\mathbf{Z}_{2}$ & $\begin{array}{l}\left\{\left[-c\left(F_{9}\right)^{2}+c\left(F_{9}\right) c\left(-F_{9}\right)\right]_{99}+\left[-c\left(F_{5}\right)^{2}+c\left(F_{5}\right) c\left(-F_{5}\right)\right]_{55}\right. \\
\left.-\left[c\left(F_{5}\right) c\left(F_{9}\right)\right]_{95}+\left[c\left(2 F_{9}\right)\right]_{9}+\left[c\left(2 F_{5}\right)\right]_{5}\right\} I_{1 / 2}\end{array}$ \\
\hline $\mathbf{Z}_{3}$ & $\begin{array}{l}\left\{\left[-\frac{1}{2} c\left(F_{9}^{a}\right)^{2}+\frac{1}{2} c\left(F_{9}\right)^{2}+c\left(F_{9}^{a}\right) c\left(-F_{9}^{a}\right)-c\left(F_{9}^{b}\right) c\left(-F_{9}^{b}\right)\right]_{99}\right. \\
\left.+\frac{1}{2}\left[c\left(2 F_{9}^{a}\right)-c\left(2 F_{9}^{b}\right)\right]_{9}\right\} I_{1 / 2}\end{array}$ \\
\hline $\mathbf{Z}_{4}$ & $\begin{array}{l}\left\{\left[-\frac{1}{2}\left(c\left(F_{9}^{a}\right)+c\left(F_{9}^{b}\right)\right)^{2}+c\left(F_{9}^{a}\right) c\left(-F_{9}^{a}\right)+c\left(F_{9}^{b}\right) c\left(-F_{9}^{b}\right)\right]_{99}\right. \\
+\left[-\frac{1}{2}\left(c\left(F_{5}^{a}\right)+c\left(F_{5}^{b}\right)\right)^{2}+c\left(F_{5}^{a}\right) c\left(-F_{5}^{a}\right)+c\left(F_{5}^{b}\right) c\left(-F_{5}^{b}\right)\right]_{55} \\
-\left[c\left(F_{9}^{a}\right) c\left(F_{5}^{a}\right)+c\left(F_{9}^{b}\right) c\left(F_{5}^{b}\right)\right]_{95} \\
\left.+\frac{1}{2}\left[c\left(2 F_{9}^{a}\right)+c\left(2 F_{9}^{b}\right)\right]_{9}+\frac{1}{2}\left[c\left(2 F_{5}^{a}\right)+c\left(2 F_{5}^{b}\right)\right]_{5}\right\} I_{1 / 2}\end{array}$ \\
\hline $\mathbf{Z}_{6}$ & $\begin{aligned}\{ & {\left[-\frac{1}{2} c\left(F_{9}^{a}\right)^{2}-\frac{1}{2} c\left(F_{9}^{b}\right)^{2}-\left(c\left(F_{9}^{a}\right)+c\left(F_{9}^{b}\right)\right) c\left(F_{9}^{c}\right)\right.} \\
& \left.+c\left(F_{9}^{a}\right) c\left(-F_{9}^{a}\right)+c\left(F_{9}^{b}\right) c\left(-F_{9}^{b}\right)+c\left(F_{9}^{c}\right) c\left(-F_{9}^{c}\right)\right]_{99} \\
+ & {\left[-\frac{1}{2} c\left(F_{5}^{a}\right)^{2}-\frac{1}{2} c\left(F_{5}^{b}\right)^{2}-\left(c\left(F_{5}^{a}\right)+c\left(F_{5}^{b}\right)\right) c\left(F_{5}^{c}\right)\right.} \\
& \left.+c\left(F_{5}^{a}\right) c\left(-F_{5}^{a}\right)+c\left(F_{5}^{b}\right) c\left(-F_{5}^{b}\right)+c\left(F_{5}^{c}\right) c\left(-F_{5}^{c}\right)\right]_{55} \\
- & {\left[c\left(F_{9}^{a}\right) c\left(F_{5}^{a}\right)+c\left(F_{9}^{b}\right) c\left(F_{5}^{b}\right)+c\left(F_{9}^{c}\right) c\left(F_{5}^{c}\right)\right]_{95} } \\
+ & \left.\frac{1}{2}\left[c\left(2 F_{9}^{a}\right)+c\left(2 F_{9}^{b}\right)\right]_{9}+\frac{1}{2}\left[c\left(2 F_{5}^{a}\right)+c\left(2 F_{5}^{b}\right)\right]_{5}\right\} I_{1 / 2}\end{aligned}$ \\
\hline
\end{tabular}
polynomials for all the models are reported in the table below. 
We have used the fact that in six dimensions only terms with an even number of curvatures can appear. For later convenience, we do not expand explicitly the anomaly polynomials, but it is understood that only the 8-form component of the quoted expressions is relevant. The Latin letters $a, b, c, \ldots$ label the gauge group factors in the same order as they appear in the previous table. The terms in the square brackets with index 99, 55 and 95 are expected to be canceled by inflows associated to the D-brane-D-brane interaction encoded in the annulus amplitude in the 99, 55 and 95 sectors. Similarly, the terms in the square brackets with index 9 and 5 are expected to be canceled by the inflows associated to the D-brane-fixed-planes interaction encoded in the Möbius strip amplitude in the 9 and 5 sectors.

Both D-branes (B) and fixed-points (F) are involved in the inflow mechanism. The annulus, Möbius strip and Klein bottle amplitudes in the odd spin-structure encode the anomaly inflow arising from the RR magnetic interaction between two D-branes (BB), a D-brane and a fixed-point (BF), and two fixed-points (FF) respectively. Since there are three types of interaction among only two kinds of objects, the factorization of these interactions is non-trivial. The total inflow induced by each surface is obtained by summing over all the sectors of the orientifold group the partition function of a quantum mechanical model in the (six-dimensional) odd spin-structure.

In the operatorial formalism, the relevant odd spin-structure amplitudes that we have to compute on the annulus, Möbius strip and Klein bottle, are given by the following partition functions:

$$
\begin{aligned}
& Z_{A}=\frac{1}{4 N} \sum_{k=0}^{N-1} \operatorname{Tr}_{R}\left[g^{k}(-1)^{F} e^{-t H(R, F)}\right] \\
& Z_{M}=\frac{1}{4 N} \sum_{k=0}^{N-1} \operatorname{Tr}_{R}\left[\Omega g^{k}(-1)^{F} e^{-t H(R, F)}\right] \\
& Z_{K}=\frac{1}{8 N} \sum_{k=0}^{N-1} \sum_{m=0}^{N-1} \operatorname{Tr}_{R R}^{(m)}\left[\Omega g^{k}(-1)^{F+\tilde{F}} e^{-t H(R, F)}\right],
\end{aligned}
$$

where a sum over Chan-Paton indices is understood for the case of the annulus and Möbius strip surfaces. In the Klein bottle amplitude, the extra sum includes all the closed string twisted sectors. The overall factors come form the $\Omega$ and GSO projections. In the following we will not present the details of the evaluation of the partition functions of the quantum mechanical models that are reported in [6, 目].

\section{Annulus}

Consider first the various annulus amplitudes. The 99 contribution reads:

$$
Z_{A}^{99}\left(R, F_{9}\right)=\frac{1}{4 N} \sum_{k=1}^{N-1} N_{k} Z_{k}^{B} Z_{k}^{F} \operatorname{ch}^{2}\left(\gamma_{k} F_{9}\right) \widehat{A}(R),
$$

where the Chern class

$$
\operatorname{ch}\left(\gamma_{k} F_{9}\right) \equiv \operatorname{tr}\left[\gamma_{k, 9} e^{i F_{9} / 2 \pi}\right]
$$


is defined in the Chan-Paton representation (which is built out of fundamental representations of the various factors of the gauge group) and includes the matrix representation $\gamma_{k, 9}$ of the twist $g^{k}$ induced by the orientifold group action in the 9 sector. $\widehat{A}(R)$ is the roof genus, and is unaffected by the orientifold projection. Finally, $N_{k}$ and $Z_{k}^{B, F}$ take into account the internal partition functions of the four compact bosons and fermions, which in the 99 sector have an integer mode expansion and admit zeroenergy states. The bosons have zero modes $x_{0}^{i} \sim g^{k} x_{0}^{i}$ in each $k$-twisted sector, and one has therefore to sum over all the $k$-fixed-points $\mathrm{Fk}$, whose number is given by

$$
N_{k}=\left(2 \sin \frac{\pi k}{N}\right)^{4}, k=1, \ldots, N-1 .
$$

The fermions have instead zero modes only in the untwisted sector $k=0$, leaving a vanishing partition function in this sector. Finally, $Z_{k}^{B, F}$ represent the partition functions in the $k$-twisted sector of the remaining fluctuations of the four internal bosons and fermions respectively, and are given by

$$
Z_{k}^{B}=\left(2 \sin \frac{\pi k}{N}\right)^{-4}, Z_{k}^{F}=\left(2 \sin \frac{\pi k}{N}\right)^{2}, k=1, \ldots, N-1 .
$$

In the 55 sector the Dirichlet boundary conditions still allow zero-energy states for fermions but not for bosons. The corresponding contribution is then:

$$
Z_{A}^{55}\left(R, F_{5}\right)=\frac{1}{4 N} \sum_{k=1}^{N-1} Z_{k}^{F} \operatorname{ch}^{2}\left(\gamma_{k} F_{5}\right) \widehat{A}(R)
$$

where now

$$
\operatorname{ch}\left(\gamma_{k} F_{5}\right) \equiv \operatorname{tr}\left[\gamma_{k, 5} e^{i F_{5} / 2 \pi}\right] .
$$

Finally, in the 95 sector the internal fields satisfy mixed Neumann-Dirichlet boundary conditions and have a half-integer mode expansion. This implies that no zero-energy states are present in these sectors and correspondingly

$$
Z_{A}^{95}\left(R, F_{9}, F_{5}\right)=-\frac{1}{2 N} \sum_{k=0}^{N-1} \operatorname{ch}\left(\gamma_{k} F_{9}\right) \operatorname{ch}\left(\gamma_{k} F_{5}\right) \widehat{A}(R) .
$$

The minus sign in (3.12) is due to the fact that the 95 Ramond vacuum differs by one unit of fermionic charge with respect to the 99 and 55 Ramond vacua, and the factor two takes into account the two orientations 95 and 59. From the closed string channel point of view, these expressions encode the D9-D9, D5-D5 and D5-D9 magnetic interactions. The $k$-th term in each sum corresponds to the exchange of RR forms of the $k$-th twisted sector.

\section{Möbius strip}

The Möbius strip contribution in the 9 sector reads

$$
I_{M}^{9}\left(R, F_{9}\right)=-\frac{1}{4 N} \sum_{k=1}^{N-1} N_{k} Z_{k}^{B} Z_{k}^{F} \operatorname{ch}\left(\gamma_{\Omega k}^{-1} \gamma_{\Omega k}^{T} 2 F_{9}\right) \widehat{A}(R),
$$


where again we have taken into account the twist induced by the orientifold group action on the Chern class. The factor two entering in the Chern character is due to the fact that the boundary of the Möbius strip is twice longer than one of the two boundaries of the annulus. The analysis for the four internal directions is identical to that presented for the 99 sector of the annulus and the corresponding contribution is identical and given by (3.9). In the 5 sector we get instead

$$
I_{M}^{5}\left(R, F_{5}\right)=-\frac{1}{4 N} \sum_{k=0}^{N-1} Z_{k+N / 2}^{F} \operatorname{ch}\left(\gamma_{\Omega k}^{-1} \gamma_{\Omega k}^{T} 2 F_{5}\right) \widehat{A}(R),
$$

where again the four fermions present zero-energy states, absent for the bosons. The extra $N / 2$-twist is needed to implement Dirichlet boundary conditions in the Möbius strip.

In the closed string channel, these expressions correspond to the D9-Fk and D5Fk magnetic interactions. The $k$-th term in each sum involves $k$-fixed-points and corresponds to the exchange of RR forms in the $2 k$-th twisted sector. Correspondingly, the following relations hold (see [21])

$$
\operatorname{ch}\left(\gamma_{\Omega k}^{-1} \gamma_{\Omega k}^{T} 2 F\right)= \pm \operatorname{ch}\left(\gamma_{2 k} 2 F\right),
$$

the + and - signs holding in the 9 and 5 sectors respectively.

\section{Klein bottle}

In the closed string sector, $\Omega$ exchanges the $m$-th twisted sector with the $(N-m)$ th. This implies that the only sectors which contributes to the Klein bottle partition function in (3.5) are the untwisted sector $m=0$ and, for $N$ even, the middle sector $m=N / 2$. We then get two distinct contributions. The one coming from the untwisted $m=0$ sector is given by:

$$
I_{K}(R)=\frac{1}{16 N} \sum_{k=1}^{N-1} N_{k+N / 2} Z_{k+N / 2}^{B} Z_{k}^{F} Z_{k+N / 2}^{F} \widehat{L}(R),
$$

where we have taken into account that in this sector, both the internal bosons and and fermions have zero-energy states and that, analogously to the Möbius strip case in the 5 sector, an extra $N / 2$-twist is needed to implement the correct crosscap conditions. Moreover, in this surface left-moving and right-moving fermions are not simply identified but split into two combinations with periodic and anti-periodic zero-energy states respectively [6]. This explains the various factors entering in (3.16).

In the $N / 2$-twisted sector, the only zero-energy states are the bosonic zero modes $x_{0}^{i} \sim-x_{0}^{i}$. Since in (3.5) we have also a sum over all the $g^{k}$-twists we get:

$$
I_{K}^{\prime}(R)=-\frac{1}{16 N} \widehat{L}(R) \sum_{k=0}^{N-1} N_{k}^{\prime},
$$

\footnotetext{
${ }^{6}$ Notice that $\Omega$ reported here does not precisely coincide with the IIB world-sheet parity operator. See 23 for a discussion about this point.
} 
where $N_{k}^{\prime}$ represents the number of $N / 2$-fixed-points that are also $k$-fixed. These numbers are given by $N_{0}^{\prime}=N_{N / 2}^{\prime}=16$, and $N_{k}^{\prime}=N_{N-k}^{\prime}$ with

$$
N_{k}^{\prime}=\min \left\{N_{k}, N_{k+N / 2}\right\}, k=1, \ldots, N / 2-1 .
$$

The minus sign in (3.17) arises from the action of $\Omega$ on the $N / 2$-twisted vacua.

From the closed string channel point of view, these two contributions correspond respectively to the $\mathrm{Fk}-\mathrm{Fk}$ and $\mathrm{Fk}-\mathrm{Fk}+N / 2$ magnetic interactions, and the $k$-th term in each sum involves the exchange of RR forms in the $2 k$-th twisted sector. $\widehat{L}(R)$ indicates the Hirzebruch polynomial, and is crucial for the cancellation of anomalies due to (anti)self-dual tensors [6].

\section{Inflows}

Collecting these results, one finds the following expressions for the various inflows:

$$
\begin{aligned}
& I_{B B}^{99,55}\left(R, F_{9,5}\right)=\frac{1}{4 N} \sum_{k=1}^{N-1}\left(2 \sin \frac{\pi k}{N}\right)^{2} \operatorname{ch}^{2}\left(\gamma_{k} F_{9,5}\right) I_{1 / 2}(R) \\
& I_{B B}^{95}\left(R, F_{9}, F_{5}\right)=-\frac{1}{2 N} \sum_{k=0}^{N-1} \operatorname{ch}\left(\gamma_{k} F_{9}\right) \operatorname{ch}\left(\gamma_{k} F_{5}\right) I_{1 / 2}(R) \\
& I_{B F}^{9}\left(R, F_{9}\right)=-\frac{1}{4 N} \sum_{k=1}^{N-1}\left(2 \sin \frac{\pi k}{N}\right)^{2} \operatorname{ch}\left(\gamma_{2 k} 2 F_{9}\right) I_{1 / 2}(R) \\
& I_{B F}^{5}\left(R, F_{5}\right)=\frac{1}{4 N} \sum_{k=0}^{N-1}\left(2 \cos \frac{\pi k}{N}\right)^{2} \operatorname{ch}\left(\gamma_{2 k} 2 F_{5}\right) I_{1 / 2}(R), \\
& I_{F F}(R)=-\frac{1}{2 N} \sum_{k=0}^{N-1}\left[\left(2 \sin \frac{2 \pi k}{N}\right)^{2}-N_{k}^{\prime}\right] I_{A}(R) .
\end{aligned}
$$

In order to compute explicitly these inflows and compare them to the anomalies in the spectrum, one needs the explicit representation of the $\gamma_{k}$ matrices. In a suitable basis, one can choose $\gamma_{k, 9}=(\gamma)^{k}, \gamma_{k, 5}=\left(\gamma^{*}\right)^{k}, \gamma^{*}=\gamma^{-1}$, with $\gamma$ given in the following tablet

\begin{tabular}{|c|c|}
\hline Model & $\gamma$ \\
\hline $\mathbf{Z}_{2}$ & $\operatorname{diag}\left(e^{\frac{\pi}{2} i} \mathbf{I}_{\mathbf{1 6}}, e^{-\frac{\pi}{2} i} \mathbf{I}_{\overline{\mathbf{1 6}}}\right)$ \\
\hline $\mathbf{Z}_{3}$ & $\operatorname{diag}\left(e^{\frac{2 \pi}{3} i} \mathbf{I}_{\mathbf{8}}{ }^{a}, e^{-\frac{2 \pi}{3} i} \mathbf{I}_{\mathbf{8}}{ }^{a}, \mathbf{I}_{\mathbf{1 6}}{ }^{b}\right)$ \\
\hline $\mathbf{Z}_{4}$ & $\operatorname{diag}\left(e^{\frac{\pi}{4} i} \mathbf{I}_{\mathbf{8}}{ }^{a}, e^{-\frac{\pi}{4} i} \mathbf{I}_{\overline{\mathbf{8}}}{ }^{a}, e^{-\frac{3 \pi}{4} i} \mathbf{I}_{\mathbf{8}}{ }^{b}, e^{\frac{3 \pi}{4} i} \mathbf{I}_{\mathbf{8}}{ }^{b}\right)$ \\
\hline $\mathbf{Z}_{6}$ & $\operatorname{diag}\left(e^{\frac{\pi}{6} i} \mathbf{I}_{\mathbf{4}}{ }^{a}, e^{-\frac{\pi}{6} i} \mathbf{I}_{\overline{\mathbf{4}}}{ }^{a}, e^{\frac{5 \pi}{6} i} \mathbf{I}_{\mathbf{4}}{ }^{b}, e^{-\frac{5 \pi}{6} i} \mathbf{I}_{\mathbf{4}}{ }^{b}, e^{-\frac{\pi}{2} i} \mathbf{I}_{\mathbf{8}}{ }^{c}, e^{\frac{\pi}{2} i} \mathbf{I}_{\overline{\mathbf{8}}}{ }^{c}\right)$ \\
\hline
\end{tabular}

\footnotetext{
${ }^{7}$ The matrices used here differ from those in [21] in the fact that there is no relative phase between the matrices in the 9 and the 5 sectors. The necessity of such a phase is avoided here by taking explicitly into account the fixed-point degeneracy, achieving a complete symmetry between the 9 and the 5 sectors.
} 
Latin letters again refer to the various factors of the gauge group, and $\mathbf{I}_{\rho}$ indicates the identity in the representation $\rho$.

It is straightforward but tedious to check that the total inflow, obtained by summing all the contributions above, is indeed equal to the total one-loop anomaly $I_{n}+I_{c}$, for each model. Pictorially, the BB, BF and FF parts of the inflow receive the following non-vanishing contributions

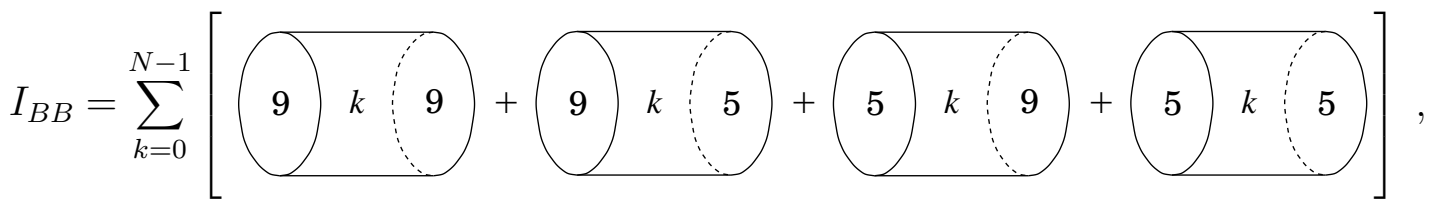

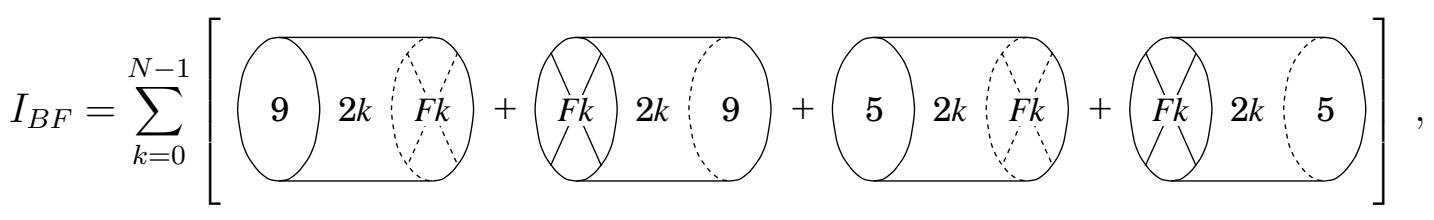

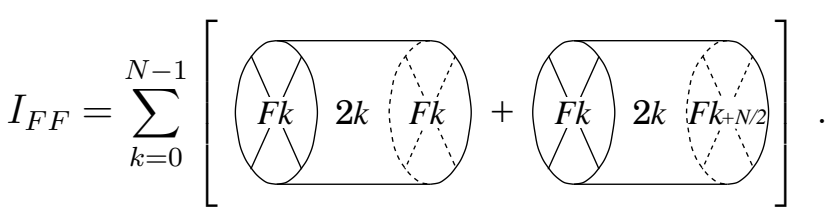

\section{Factorization of the couplings}

As discussed in previous section, the various tree-level BB, BF and FF inflows cancel separately corresponding pieces of the total one-loop anomaly, and are interpreted as magnetic interactions. Having computed all the inflows, the anomalous couplings to RR fields can be obtained by factorization. Starting from (3.19)-(3.23) and knowing which (and how) massless fields arise in these orientifold models [21] in all closed string sectors, untwisted and twisted, it is possible to identify those responsible for the inflows above. Using the crucial property [7]

$$
\sqrt{\widehat{A}(R)} \sqrt{\widehat{L}(R / 4)}=\widehat{A}(R / 2),
$$

and making use of the fact that only the 8-form components of all the polynomials are relevant to perform some rescalings, it is straightforward to show that the inflows (3.19)-(3.23) can be indeed consistently factorized.

Recall now that these inflows of anomaly are associated with RR magnetic interactions. The latter arise in string theory in the odd spin-structure, the insertion of $(-1)^{F}$ acting as Hodge duality on the propagating field [38]. It is easy to deduce from the analysis of [20, 21] that the only fields mediating RR magnetic interactions are 2forms in the RR untwisted sector, and 0-forms (scalars) magnetically dual to 4-forms, as well as anti-self-dual 2-forms, in the twisted RR sectors. The content of this kind of fields of the various models is the following. In the untwisted RR sector, there 
are always an anti-self-dual and a self-dual 2-form. They lie in the universal tensor multiplet and in the gravitational multiplet respectively, and together they form an unconstrained 2-form $b_{\mu \nu}$. In the twisted RR sectors, a variable number of scalars and anti-self-dual 2-forms occurs, depending on the model. More precisely, one has to distinguish between $k$-twisted closed string sectors with $k=N / 2$ and $k \neq N / 2$. At each of the $N_{N / 2}=16 N / 2$-fixed points, one gets a scalar $\varphi$ belonging to an extra hypermultiplet. Some among these are further identified by the $\mathbf{Z}_{N}$ projection, and the number of independent scalars is 16 for $\mathbf{Z}_{2}, 0$ for $\mathbf{Z}_{3}, 10$ for $\mathbf{Z}_{4}$ and 6 for $\mathbf{Z}_{6}$. Similarly, at each of the $N_{k} k$-fixed-points with $k \neq N / 2$, one gets a scalar $\phi^{(k)}$, belonging to an extra hypermultiplet, and one anti-self-dual 2 -form $b_{\mu \nu}^{(k)}$, belonging to one extra tensor multiplet. Actually, these arise by combining the sectors with twists $k$ and $N-k$, which are related by the $\Omega$-projection. Again, the $\mathbf{Z}_{N}$ projection further identifies some of these among each other; the net number of them is 0 for $\mathbf{Z}_{2}, 9$ (from $k=1,2$ ) for $\mathbf{Z}_{3}, 4$ (from $k=1,3$ ) for $\mathbf{Z}_{4}$ and $1+5=6$ (from $k=1,5$ and $k=2,4$ respectively) for $\mathbf{Z}_{6}$. There is an evident and important distinction arising between the inflows due to the exchange of tensor and scalar fields. Tensor fields can have anomalous couplings only to 4 -forms, and the corresponding contribution to the 8-form anomaly polynomial will factorize into the product of two 4-forms. Scalar fields can instead have anomalous couplings to 2 and 6-forms, and the corresponding contribution to the 8-form anomaly polynomial will factorize into the product of a 2 -form and a 6 -form. This implies that all the inflows induced by exchange of scalars and their dual 4-forms involve always the Abelian part of the gauge group.

Throughout this section, for simplicity, we will always write at the same time anomalous couplings for forms and their duals, despite the well known fact that there is no local and covariant Lagrangian in which a potential and its dual can appear at the same time. More precisely, one should write the couplings to the dual potential as corrections to the kinetic terms for the field strength of the original potential, modifying then its Bianchi identity. In presence of anti-self-dual forms our formulae for the couplings should be considered as formal expressions that reproduce the right inflow of anomaly. More precisely, they should be written either in a noncovariant way, by using the electro-magnetically symmetric action introduced in [39], or covariantly using the formalism introduced in [40].

We now present a general analysis, valid for all the K3 orientifolds considered here, allowing to extract in a systematic way the couplings and to emphasize some general features which are common to all models. In order to keep the analysis as general as possible, we find it convenient to keep all the $16 \mathbf{Z}_{2}$-twisted scalars and the $N_{k}$ tensors and scalars arising from the $k$ and $N-k$ twisted sectors, as independent states. Although the physical propagating states are less than that, as mentioned, this will facilitate the general analysis and yet remain correct. In next section, the anomalous couplings associated to the physical scalar fields will be explicitly displayed.

In order to figure out which states are responsible for the various inflows, recall 
that in (3.19)-(3.20) the insertion of $g^{k}$ acts as a $k$-twist in $\sigma$ for the closed string state exchanged and as a $2 k$-twist in $\sigma$ in (3.21)-(3.23). It is natural to consider separately the $k=0, k=N / 2$ closed string sectors which are special in many respects, and to group the remaining closed string sectors into conjugate pairs, adding the contributions of the $k$ and $N-k$ twisted sectors which correspond to the same intermediate closed string states.

\section{Untwisted closed string exchange}

The non-vanishing contributions to the inflows (3.19)-(3.23) involving untwisted closed string states of the $k=0$ sector, come form the terms $k=0$ in (3.20) and (3.22), $k=N / 2$ in (3.21) and $k=0, N / 2$ in (3.23). They can be written as

$$
\begin{aligned}
& I_{B B}^{(0) 95}\left(R, F_{5}, F_{9}\right)=-Y_{(0)}^{9}\left(F_{9}, R\right) Y_{(0)}^{5}\left(F_{5}, R\right) \\
& I_{B F}^{(0) 9,5}\left(R, F_{9,5}\right)=-Y_{(0)}^{9,5}\left(F_{9,5}, R\right) Y_{(0)}(R) \\
& I_{F F}^{(0)}(R)=-Y_{(0)}(R) Y_{(0)}(R)
\end{aligned}
$$

where

$$
\begin{aligned}
& Y_{(0)}^{5}\left(F_{5}, R\right)=\frac{1}{\sqrt{2 N}} \operatorname{ch}\left(F_{5}\right) \sqrt{\widehat{A}(R)} \\
& Y_{(0)}^{9}\left(F_{9}, R\right)=\frac{1}{\sqrt{2 N}} \operatorname{ch}\left(F_{9}\right) \sqrt{\widehat{A}(R)} \\
& Y_{(0)}(R)=-\frac{32}{\sqrt{2 N}} \sqrt{\widehat{L}(R / 4)}
\end{aligned}
$$

The RR fields involved in the inflow are the untwisted 2-form $b_{\mu \nu}$ and its magnetic dual $\tilde{b}_{\mu \nu}$. Form a ten-dimensional point of view, $b_{\mu \nu}$ is the trivial dimensional reduction of the RR 2-form, whereas $\tilde{b}_{\mu \nu}$ arises from integrating the RR 6 -form on $T^{4}$. The magnetic duality of $b_{\mu \nu}$ and $\tilde{b}_{\mu \nu}$ in six dimensions follows from the fact that the RR 2 and 6 -forms in ten-dimensions have field-strengths which are Hodge dual to each other. Form the known form of the anomalous couplings of D-branes and O-planes in ten-dimensions, we then expect that the combinations of fields coupling to D5-branes and O5-planes, and D9-branes and O9-planes, are simply:

$$
C_{(0)}=b, \quad \tilde{C}_{(0)}=\tilde{b}
$$

The reason for this is the following. D5-branes and O5-planes couple to both the RR 2-form and 6-form in ten-dimension. However, the total charge with respect to the latter cancels by the requirement of tadpole cancellation, and only the 2-form is relevant, producing $b$ in six dimensions. Conversely, since all the internal curvatures vanish, the integral over $T^{4}$ in the anomalous couplings of D9-branes and O9-planes can act only on the RR 6-form, producing $\tilde{b}$ in six dimensions. 
Using the results of appendix A, it is then straightforward to show that the anomalous couplings

$$
\begin{aligned}
& S_{D 5}^{(0)}=\sqrt{2 \pi} \int C_{(0)} \wedge Y_{(0)}^{5}\left(F_{5}, R\right), \\
& S_{D 9}^{(0)}=\sqrt{2 \pi} \int \tilde{C}_{(0)} \wedge Y_{(0)}^{9}\left(F_{9}, R\right), \\
& S_{O 5}^{(0)}=\sqrt{2 \pi} \int C_{(0)} \wedge Y_{(0)}(R), \\
& S_{O 9}^{(0)}=\sqrt{2 \pi} \int \tilde{C}_{(0)} \wedge Y_{(0)}(R),
\end{aligned}
$$

reproduce the above inflows.

\section{$\mathrm{N} /$ 2-twisted closed string exchange}

The non-vanishing contributions to the inflows (3.19)-(3.23) involving $\mathbf{Z}_{2}$-twisted closed string states of the $k=N / 2$ sector, come from the $k=N / 2$ terms in (3.19) and 3.20 . They can be written as

$$
\begin{aligned}
& I_{B B}^{(N / 2) 99}\left(R, F_{9}\right)=\frac{1}{2} N_{N / 2} Y_{(N / 2)}^{9}\left(F_{9}, R\right) Y_{(N / 2)}^{9}\left(F_{9}, R\right) \\
& I_{B B}^{(N / 2) 55}\left(R, F_{5}\right)=\frac{1}{2} Y_{(N / 2)}^{5}\left(F_{5}, R\right) Y_{(N / 2)}^{5}\left(F_{5}, R\right) \\
& I_{B B}^{(N / 2) 95}\left(R, F_{5}, F_{9}\right)=Y_{(N / 2)}^{9}\left(F_{9}, R\right) Y_{(N / 2)}^{5}\left(F_{5}, R\right)
\end{aligned}
$$

where $N_{N / 2}=16$ and

$$
\begin{aligned}
& Y_{(N / 2)}^{5}\left(F_{5}, R\right)=-\frac{1}{\sqrt{2 N}} 2 \operatorname{ch}\left(\gamma_{N / 2} F_{5}\right) \sqrt{\widehat{A}(R)} \\
& Y_{(N / 2)}^{9}\left(F_{9}, R\right)=\frac{1}{\sqrt{2 N}} 2^{-1} \operatorname{ch}\left(\gamma_{N / 2} F_{9}\right) \sqrt{\widehat{A}(R)} .
\end{aligned}
$$

The RR states responsible for the inflow are the scalars $\varphi^{i}$ and their magnetically dual 4 -forms $\tilde{\varphi}^{i}$, with $i=1, \ldots, N_{N / 2}$. To produce the correct kind of inflows, the anomalous couplings have to involve the combinations

$$
C_{(N / 2)}^{i}=\varphi^{i}+\tilde{\varphi}^{i}
$$

The required anomalous couplings are then found to be

$$
\begin{aligned}
S_{D 5}^{(N / 2)} & =\sqrt{2 \pi} \int C_{(N / 2)}^{1} \wedge Y_{(N / 2)}^{5}\left(F_{5}, R\right), \\
S_{D 9}^{(N / 2)} & =\sqrt{2 \pi} \sum_{i=1}^{N_{N / 2}} \int C_{(N / 2)}^{i} \wedge Y_{(N / 2)}^{9}\left(F_{9}, R\right),
\end{aligned}
$$

where $i=1$ refers to the fixed-point at the origin, where all the D5-branes sit. Further details are reported in appendix A.

${ }^{8}$ There is also a potential contribution from the $k=N / 4$ term in the BF and FF inflows (3.21)(3.23). This exists only for the $\mathbf{Z}_{4}$ model and happens to cancel out. 


\section{Twisted closed string exchange $(\mathrm{k} \neq \mathbf{0}, \mathrm{N} / \mathbf{2})$}

In order to extract the inflow associated to $k$ and $N-k$-twisted states, we first group the $k$-th and $N-k$-th term of each sum in the inflows (3.19)-(3.23). The result can be rewritten as a sum over $k=1, \ldots, N / 2-1$ only, with the value $2 k=N / 2$ excluded for the $\mathrm{BF}$ and $\mathrm{FF}$ inflows, of the following expressions?

$$
\begin{aligned}
& I_{B B}^{(k) 99}\left(R, F_{9}\right)=\frac{1}{2} N_{k} Y_{(k)}^{9}\left(F_{9}, R\right) Y_{(k)}^{9}\left(F_{9}, R\right), \\
& I_{B B}^{(k) 55}\left(R, F_{5}\right)=\frac{1}{2} Y_{(k)}^{5}\left(F_{5}, R\right) Y_{(k)}^{5}\left(F_{5}, R\right), \\
& I_{B B}^{(k) 95}\left(R, F_{5}, F_{9}\right)=Y_{(k)}^{9}\left(F_{9}, R\right) Y_{(k)}^{5}\left(F_{5}, R\right), \\
& I_{B F}^{(2 k) 9}\left(R, F_{9}\right)=N_{k} Y_{(2 k)}^{9}\left(F_{9}, R\right) Y_{(2 k)}(R), \\
& I_{B F}^{(2 k) 5}\left(R, F_{5}\right)=Y_{(2 k)}^{5}\left(F_{5}, R\right) Y_{(2 k)}(R), \\
& I_{F F}^{(2 k)}(R)=\frac{1}{2}\left[N_{k} Y_{(2 k)}(R) Y_{(2 k)}(R)+N_{k}^{\prime} Y_{(2 k)}(R) Y_{(2 k+N)}(R)\right],
\end{aligned}
$$

where

$$
\begin{aligned}
& Y_{(k)}^{5}\left(F_{5}, R\right)=-\frac{1}{\sqrt{N}}\left(2 \sin \frac{\pi k}{N}\right) \operatorname{ch}\left(\gamma_{k} F_{5}\right) \sqrt{\widehat{A}(R)}, \\
& Y_{(k)}^{9}\left(F_{9}, R\right)=\frac{1}{\sqrt{N}}\left(2 \sin \frac{\pi k}{N}\right)^{-1} \operatorname{ch}\left(\gamma_{k} F_{9}\right) \sqrt{\widehat{A}(R)}, \\
& Y_{(2 k)}(R)=-\frac{8}{\sqrt{N}} \cot \frac{\pi k}{N} \sqrt{\widehat{L}(R / 4)} .
\end{aligned}
$$

For each $k=1, \ldots, N / 2-1$, the BB inflows in (4.14) are due the exchange of $k$-twisted closed string states, whereas the $\mathrm{BF}$ and $\mathrm{FF}$ inflows are due to the exchange of $2 k$ twisted closed string states $(2 k \neq N / 2)$. More precisely, recall that the RR states responsible for the inflow in a generic $k \neq 0, N / 2$ sector are the scalars $\phi_{(k)}^{i_{k}}$ and their magnetic dual 4-forms $\tilde{\phi}_{(k)}^{i_{k}}$, as well as the 2-forms $b_{(k)}^{i_{k}}$ and their dual 2-forms $\tilde{b}_{(k)}^{i_{k}}$, with $i_{k}=1, \ldots, N_{k}$. As shown in appendix A, in order to produce the correct kind of inflows, the anomalous couplings have to involve the combinations

$$
C_{(k)}^{i_{k}}=\phi_{(k)}^{i_{k}}+\tilde{\phi}_{(k)}^{i_{k}}+\frac{1}{\sqrt{2}}\left(b_{(k)}^{i_{k}}-\tilde{b}_{(k)}^{i_{k}}\right) .
$$

The required anomalous couplings are then given by

$$
\begin{aligned}
& S_{D 5}^{(k)}=\sqrt{2 \pi} \int C_{(k)}^{1} \wedge Y_{(k)}^{5}\left(F_{5}, R\right), \\
& S_{D 9}^{(k)}=\sqrt{2 \pi} \sum_{i_{k}=1}^{N_{k}} \int C_{(k)}^{i_{k}} \wedge Y_{(k)}^{9}\left(F_{9}, R\right), \\
& S_{F k}^{(2 k)}=\sqrt{2 \pi} \sum_{i_{k}=1}^{N_{k}} \int C_{(2 k)}^{i_{k}} \wedge Y_{(2 k)}(R),
\end{aligned}
$$

\footnotetext{
${ }^{9}$ It is understood that one has to take the integer part of $N / 2-1$.
} 
for $k=1, \ldots, N / 2-1$, with $k \neq N / 4$ in (4.19). Again $i_{k}=1$ refers to the fixedpoint at the origin, where all the D5-branes sit. Notice that the fields $C_{(2 k)}$ with $2 k>N / 2$ in (4.19) (actually arising only for the $F 2$ fixed-point in the $\mathbf{Z}_{6}$ model) are not independent fields, but rather are defined as $C_{(2 k)}=\tilde{C}_{(N-2 k)}$ in terms of those with $2 k<N / 2$. This is at the origin of the second term in the Klein bottle contribution of (4.14). Again, some details needed to check that these anomalous couplings reproduce the above inflows are reported in appendix A.

Summarizing, the anomalous couplings for D5-branes, D9-branes and Fk-fixedpoints, in a generic $D=6 \mathbf{Z}_{N}$ orientifold model, are given by equations (4.5)-(4.8) in the untwisted sector, (4.12) and (4.13) in the $\mathbf{Z}_{2}$-twisted sector, and (4.17)-(4.19) in the other twisted sectors. As usual, it is understood that only the appropriate component of the polynomials has to be considered, in this case the 6 -form component. Notice that the inflow completely fixes the coefficients in the combinations (4.11) and (4.16) of the RR tensors, since they are anti-self dual, but strictly speaking it does not fix completely those between the RR scalars and dual four-forms. Our choice is just the simplest and most natural one in which they are taken to be equal. This should be always taken into account in the next sections when we write explicitly these couplings. Not surprisingly, D5 and D9-branes have RR anomalous couplings in each closed string sector. O5-planes, corresponding in our language to the $\mathbf{Z}_{2^{-}}$ fixed-points $F_{N / 2}$, as well as O9-planes, have instead RR anomalous couplings only in the untwisted sector. Finally, the other fixed-points $F k$ have non-vanishing RR anomalous couplings only in the $2 k$-twisted sector. The explicit results for each of the models we are considering are reported in appendix B. Notice finally that for $k=0$ and $k=N / 2$, the charges contain only $4 p$-forms and $4 p+2$-forms respectively, whereas for $k \neq 0, N / 2$, they contain generically $2 p$-forms. In the anomalous couplings above, this is compatible with the fact that the formal sums $C_{i_{k}}^{(k)}$ contain only 2 -forms for $k=0$, only 0 and 4 -forms for $k=N / 2$, and both 0,2 and 4 -forms for $k \neq 0, N / 2$.

\section{Anomaly cancellation}

In this section, we present a more detailed model by model analysis of the inflow mechanism. We first identify more precisely which $\mathrm{RR}$ fields give rise to the various sub-inflows. Applying the general factorization described in last section, we then write explicitly all the anomalous couplings for each model, and verify that these couplings lead to the correct inflow. This allow to find the factorized form of the anomaly and the inflow, exhibiting explicitly the details of the Green-Schwarz mechanism at work. As expected, we will see that similarly to what happens in the $\mathbf{Z}_{2}$ model [26, various $U(1)$ factors of the gauge group are spontaneously broken by a Higgs mechanism involving the various RR twisted scalars, belonging to hypermultiplets. We will discuss this phenomenon in more detail in the next section. For completeness, we report below the analysis of the $\mathbf{Z}_{2}$ model, although a full and detailed discussion 
of anomaly cancellation in this model has been already given in [26].

\section{1. $\mathrm{Z}_{2}$-model}

The RR massless states arising in the model are the following:

$$
\begin{array}{rll}
\text { Untwisted }: & b_{\mu \nu}, \quad \phi^{i}, i=1, \ldots, 6, \\
\mathbf{Z}_{2} \text {-twisted }: & \varphi^{j}, \quad j=1, \ldots, 16 .
\end{array}
$$

Each of the twisted scalars $\varphi^{j}$ belongs to a six-dimensional $N=1$ hypermultiplet. By using the general results of last section, it is straightforward to find the explicit anomalous couplings for this model, reported in (B.1). Expliciting all the polynomials, the total anomalous term is found to be

$$
\frac{L_{W Z}}{\sqrt{2 \pi}}=b X_{4}^{(5)}+\tilde{b} X_{4}^{(9)}+\varphi^{1} X_{6}^{(5)}+\tilde{\varphi}^{1} X_{2}^{(5)}+\left(\frac{1}{4} \sum_{j=1}^{16} \varphi^{j}\right) X_{6}^{(9)}+\left(\frac{1}{4} \sum_{j=1}^{16} \tilde{\varphi}^{j}\right) X_{2}^{(9)}
$$

where as usual $j=1$ refers to the fixed-point at the origin where the D5-branes sit and $(\alpha=9,5)$

$$
\begin{aligned}
& X_{4}^{(\alpha)}=\frac{1}{2(2 \pi)^{2}}\left(\frac{1}{2} \operatorname{tr} R^{2}-\operatorname{tr} F_{\alpha}^{2}\right), \\
& X_{2}^{(\alpha)}=\frac{-2}{(2 \pi)} \operatorname{tr} F_{\alpha}, \\
& X_{6}^{(\alpha)}=\frac{-1}{3(2 \pi)^{3}}\left(\frac{1}{16} \operatorname{tr} R^{2} \operatorname{tr} F_{\alpha}-\operatorname{tr} F_{\alpha}^{3}\right) .
\end{aligned}
$$

The corresponding inflow istण

$$
I=X_{4}^{(5)} X_{4}^{(9)}-X_{6}^{(5)}\left(X_{2}^{(5)}+\frac{1}{4} X_{2}^{(9)}\right)-X_{6}^{(9)}\left(X_{2}^{(9)}+\frac{1}{4} X_{2}^{(5)}\right) .
$$

This leads to an anomalous variation of the effective action that is exactly equal and opposite to the total anomaly $A=2 \pi i \int\left(I_{n}+I_{c}\right)^{(1)}$, as can be easily verified.

\section{2. $\mathrm{Z}_{3}$-model}

The massless RR states are:

$$
\begin{aligned}
& \text { Untwisted : } b_{\mu \nu}, \phi^{i}, i=1, \ldots, 4 \text {, } \\
& \mathbf{Z}_{3} \text {-twisted : } b_{\mu \nu}^{\prime m}, \phi^{\prime m}, m=1, \ldots, 9 \text {. }
\end{aligned}
$$

Each of the 9 twisted scalars belong to a hypermultiplet, whereas each of the 9 antisymmetric tensors belong to a tensor multiplet. In this case only twisted closed string states participate to the inflow. The anomalous couplings are again easily

\footnotetext{
${ }^{10}$ The minus sign between the $X_{4} X_{4}$ inflows and the $X_{6} X_{2}$ inflows is due to the conjugation of the gauge curvature in the formula for the inflow, see appendix A.
} 
found, and are reported in $(\mathbb{B . 2})$. Expliciting the polynomials, one then finds

$$
\begin{aligned}
\frac{L_{W Z}}{\sqrt{2 \pi}}= & \tilde{b} X_{4}^{(9)}+\left(\frac{1}{3} \sum_{m=1}^{9} b^{\prime m}\right) X_{4}^{\prime(9)}-\left(\frac{1}{3} \sum_{m=1}^{9} \tilde{b}^{\prime m}\right) X_{4}^{\prime(9)} \\
& +\left(\frac{1}{3} \sum_{m=1}^{9} \phi^{\prime m}\right) X_{6}^{\prime(9)}+\left(\frac{1}{3} \sum_{m=1}^{9} \tilde{\phi}^{\prime m}\right) X_{2}^{\prime(9)}
\end{aligned}
$$

where

$$
\begin{aligned}
& X_{4}^{(9)}=\frac{1}{\sqrt{6}(2 \pi)^{2}}\left[\frac{1}{2} \operatorname{tr} R^{2}-\left(\operatorname{tr} F_{9}^{a 2}+\frac{1}{2} \operatorname{tr} F_{9}^{b^{2}}\right)\right], \\
& X_{4}^{\prime(9)}=\frac{1}{2 \sqrt{2}(2 \pi)^{2}}\left(\frac{1}{4} \operatorname{tr} R^{2}+\operatorname{tr} F_{9}^{a 2}-\operatorname{tr} F_{9}^{b^{2}}\right) \\
& X_{2}^{\prime(9)}=\frac{-\sqrt{3}}{(2 \pi)} \operatorname{tr} F_{9}^{a} \\
& X_{6}^{\prime(9)}=\frac{-1}{2 \sqrt{3}(2 \pi)^{3}}\left(\frac{1}{16} \operatorname{tr} R^{2} \operatorname{tr} F_{9}^{a}-\operatorname{tr} F_{9}^{a 3}\right) .
\end{aligned}
$$

The corresponding inflow is

$$
I=-X_{4}^{\prime(9)} X_{4}^{\prime(9)}-X_{6}^{\prime(9)} X_{2}^{\prime(9)}
$$

and cancels the total anomaly polynomial $I_{n}+I_{c}$. Notice that the untwisted sector anomalous coupling does not contribute to the inflow, but gives nevertheless a non-trivial contribution to the Bianchi identity of the antisymmetric tensor of the untwisted sector.

\section{3. $\mathrm{Z}_{4}$-model}

The RR massless states are:

$$
\begin{aligned}
& \text { Untwisted : } b_{\mu \nu}, \phi^{i}, i=1, \ldots, 4 \text {, } \\
& \mathbf{Z}_{4} \text {-twisted : } b_{\mu \nu}^{\prime m}, \phi^{\prime m}, m=1, \ldots, 4 \text {, } \\
& \mathbf{Z}_{2} \text {-twisted }: \varphi^{j}, j=1, \ldots, 10 \text {. }
\end{aligned}
$$

The RR $\mathbf{Z}_{4}$-twisted scalars and tensors belong respectively to 4 hyper multiplets and 4 tensor multiplets, whereas the $\mathbf{Z}_{2}$-twisted scalars belong to 10 hypermultiplets. Using the generic results found before, one arrives to the anomalous couplings (B.3), which lead to the following anomalous Lagrangian

$$
\begin{aligned}
\frac{L_{W Z}}{\sqrt{2 \pi}}= & b X_{4}^{(5)}+\tilde{b} X_{4}^{(9)}-b^{\prime 1} X_{4}^{\prime(5)}+\tilde{b}^{\prime 1} X_{4}^{\prime(5)}+\left(\frac{1}{2} \sum_{m=1}^{4} b^{\prime m}\right) X_{4}^{\prime(9)}-\left(\frac{1}{2} \sum_{m=1}^{4} \tilde{b}^{\prime m}\right) X_{4}^{\prime(9)} \\
& +\phi^{\prime 1} X_{6}^{\prime(5)}+\tilde{\phi}^{\prime 1} X_{2}^{\prime(5)}+\left(\frac{1}{2} \sum_{m=1}^{4} \phi^{\prime m}\right) X_{6}^{\prime(9)}+\left(\frac{1}{2} \sum_{m=1}^{4} \tilde{\phi}^{\prime m}\right) X_{2}^{\prime(9)} \\
& +\varphi^{1} X_{6}^{(5)}+\tilde{\varphi}^{1} X_{2}^{(5)}+\frac{1}{4}\left(\sum_{j=1}^{4} \varphi^{j}+\sqrt{2} \sum_{j=5}^{10} \varphi^{j}\right) X_{6}^{(9)}+\frac{1}{4}\left(\sum_{j=1}^{4} \tilde{\varphi}^{j}+\sqrt{2} \sum_{j=5}^{10} \tilde{\varphi}^{j}\right) X_{2}^{(9)}
\end{aligned}
$$


As usual, $j, m=1$ indicates the fixed-point at the origin and

$$
\begin{aligned}
& X_{4}^{(\alpha)}=\frac{1}{2 \sqrt{2}(2 \pi)^{2}}\left[\frac{1}{2} \operatorname{tr} R^{2}-\left(\operatorname{tr} F_{\alpha}^{a 2}+\operatorname{tr} F_{\alpha}^{b^{2}}\right)\right], \\
& X_{4}^{\prime(\alpha)}=\frac{-1}{2 \sqrt{2}(2 \pi)^{2}}\left(\operatorname{tr} F_{\alpha}^{a 2}-\operatorname{tr} F_{\alpha}^{b^{2}}\right), \\
& X_{6}^{(\alpha)}=\frac{-1}{3 \sqrt{2}(2 \pi)^{3}}\left[\frac{1}{16} \operatorname{tr} R^{2}\left(\operatorname{tr} F_{\alpha}^{a}+\operatorname{tr} F_{\alpha}^{b}\right)-\left(\operatorname{tr} F_{\alpha}^{a 3}+\operatorname{tr} F_{\alpha}^{b^{3}}\right)\right], \\
& X_{6}^{\prime(\alpha)}=\frac{-1}{6(2 \pi)^{3}}\left[\frac{1}{16} \operatorname{tr} R^{2}\left(\operatorname{tr} F_{\alpha}^{a}-\operatorname{tr} F_{\alpha}^{b}\right)-\left(\operatorname{tr} F_{\alpha}^{a 3}-\operatorname{tr} F_{\alpha}^{b^{3}}\right)\right], \\
& X_{2}^{(\alpha)}=\frac{-\sqrt{2}}{(2 \pi)}\left(\operatorname{tr} F_{\alpha}^{a}+\operatorname{tr} F_{\alpha}^{b}\right) \\
& X_{2}^{\prime(\alpha)}=\frac{-1}{(2 \pi)}\left(\operatorname{tr} F_{\alpha}^{a}-\operatorname{tr} F_{\alpha}^{b}\right) .
\end{aligned}
$$

The corresponding inflow is

$$
\begin{aligned}
I= & X_{4}^{(5)} X_{4}^{(9)}-X_{4}^{\prime(5)}\left(X_{4}^{\prime(5)}-\frac{1}{2} X_{4}^{\prime(9)}\right)-X_{4}^{\prime(9)}\left(X_{4}^{\prime(9)}-\frac{1}{2} X_{4}^{\prime(5)}\right) \\
& -X_{6}^{\prime(5)}\left(X_{2}^{\prime(5)}+\frac{1}{2} X_{2}^{\prime(9)}\right)-X_{6}^{\prime(9)}\left(X_{2}^{\prime(9)}+\frac{1}{2} X_{2}^{\prime(5)}\right) \\
& -X_{6}^{(5)}\left(X_{2}^{(5)}+\frac{1}{4} X_{2}^{(9)}\right)-X_{6}^{(9)}\left(X_{2}^{(9)}+\frac{1}{4} X_{2}^{(5)}\right),
\end{aligned}
$$

that is equal and opposite to the total anomaly polynomial.

The form of the $U(1)$ combinations coupling to the various scalars can be checked as follows. Whenever a $k$-twisted scalar field couples to a $U(1)$ gauge field, the corresponding two-point function on the disk, with the gauge field vertex operator on the boundary and the closed string scalar vertex operator in its interior, has to be non-vanishing. Denoting with $\lambda$ the Chan-Paton wave function associated to the two $U(1)$ gauge fields, due to the $k$-twist, the amplitude in question will be proportional to $\operatorname{tr}\left(\lambda \gamma_{k}\right)$. It is easy to see that with our choice of basis, the Chan-Paton wave functions for the $U(1)$ combinations $F_{\alpha}^{a} \mp F_{\alpha}^{b}$ are $\lambda^{1,2}=\operatorname{diag}\left(\mathbf{I}_{\mathbf{8}},-\mathbf{I}_{\mathbf{8}}, \mp \mathbf{I}_{\mathbf{8}}, \pm \mathbf{I}_{\mathbf{8}}\right)$, in both the $\alpha=9,5$ sectors. It is then clear that $\operatorname{tr}\left(\lambda^{i} \gamma_{k}\right) \sim \delta_{k}^{i}$, in agreement with the fact that the inflows due to the $k=1$ and $k=2$ twisted scalars do not mix.

\section{4. $\mathrm{Z}_{6}$-model}

The RR massless states are:

$$
\begin{array}{rll}
\text { Untwisted } & : & b_{\mu \nu}, \phi^{i}, i=1, \ldots, 4, \\
\mathbf{Z}_{6} \text {-twisted }: & b_{\mu \nu}^{\prime}, \phi^{\prime}, \\
\mathbf{Z}_{3} \text {-twisted }: & b_{\mu \nu}^{\prime \prime m}, \phi^{\prime \prime m}, m=1, \ldots, 5, \\
\mathbf{Z}_{2} \text {-twisted }: & \varphi^{j}, \quad j=1, \ldots, 6 .
\end{array}
$$

The RR $\mathbf{Z}_{6}$-twisted scalars and tensors belong to 1 hyper and 1 tensor multiplet, the $\mathbf{Z}_{3}$-twisted ones to 5 hyper and 5 tensor multiplets, and the $\mathbf{Z}_{2}$-twisted scalars to 
6 hypermultiplets. The various anomalous couplings for this model are reported in (B.4), and lead to

$$
\begin{aligned}
\frac{L_{W Z}}{\sqrt{2 \pi}}= & b X_{4}^{(5)}+\tilde{b} X_{4}^{(9)}+b^{\prime}\left(X_{4}^{\prime(9)}-X_{4}^{\prime(5)}\right)-\tilde{b}^{\prime}\left(X_{4}^{\prime(9)}-X_{4}^{\prime(5)}\right) \\
& +\phi^{\prime}\left(X_{6}^{\prime(5)}+X_{6}^{\prime(9)}\right)+\tilde{\phi}^{\prime}\left(X_{2}^{\prime(5)}+X_{2}^{\prime(9)}\right)-b^{\prime \prime 1} X_{4}^{\prime \prime(5)}+\tilde{b}^{\prime \prime 1} X_{4}^{\prime \prime(5)} \\
& +\frac{1}{3}\left(b^{\prime \prime 1}+\sqrt{2} \sum_{m=2}^{5} b^{\prime \prime m}\right) X_{4}^{\prime \prime(9)}-\frac{1}{3}\left(\tilde{b}^{\prime \prime 1}+\sqrt{2} \sum_{m=2}^{5} \tilde{b}^{\prime \prime m}\right) X_{4}^{\prime \prime(9)}+\phi^{\prime \prime 1} X_{6}^{\prime \prime(5)}+\tilde{\phi}^{\prime \prime 1} X_{2}^{\prime \prime(5)} \\
& +\frac{1}{3}\left(\phi^{\prime \prime 1}+\sqrt{2} \sum_{m=2}^{5} \phi^{\prime \prime m}\right) X_{6}^{\prime \prime(9)}+\frac{1}{3}\left(\tilde{\phi}^{\prime \prime 1}+\sqrt{2} \sum_{m=2}^{5} \tilde{\phi}^{\prime \prime m}\right) X_{2}^{\prime \prime(9)}+\varphi^{1} X_{6}^{(5)}+\tilde{\varphi}^{1} X_{2}^{(5)} \\
& +\frac{1}{4}\left(\varphi^{1}+\sqrt{3} \sum_{j=2}^{6} \varphi^{j}\right) X_{6}^{(9)}+\frac{1}{4}\left(\tilde{\varphi}^{1}+\sqrt{3} \sum_{j=2}^{6} \tilde{\varphi}^{j}\right) X_{2}^{(9)}
\end{aligned}
$$

where

$$
\begin{aligned}
& X_{4}^{(\alpha)}=\frac{1}{2 \sqrt{3}(2 \pi)^{2}}\left[\frac{1}{2} \operatorname{tr} R^{2}-\left(\operatorname{tr} F_{\alpha}^{a 2}+\operatorname{tr} F_{\alpha}^{b^{2}}+\operatorname{tr} F_{\alpha}^{c 2}\right)\right], \\
& X_{4}^{\prime(\alpha)}=\frac{-1}{4(2 \pi)^{2}}\left(\operatorname{tr} F_{\alpha}^{a 2}-\operatorname{tr} F_{\alpha}^{b^{2}}\right), \\
& X_{4}^{\prime \prime(\alpha)}=\frac{-1}{4(2 \pi)^{2}}\left[\frac{1}{4} \operatorname{tr} R^{2}+\left(\operatorname{tr} F_{\alpha}^{a 2}+\operatorname{tr} F_{\alpha}^{b^{2}}-2 \operatorname{tr} F_{\alpha}^{b^{2}}\right)\right], \\
& X_{6}^{(\alpha)}=\frac{-1}{3 \sqrt{3}(2 \pi)^{3}}\left[\frac{1}{16} \operatorname{tr} R^{2}\left(\operatorname{tr} F_{\alpha}^{a}+\operatorname{tr} F_{\alpha}^{b}+\operatorname{tr} F_{\alpha}^{c}\right)-\left(\operatorname{tr} F_{\alpha}^{a 3}+\operatorname{tr} F_{\alpha}^{b^{3}}+\operatorname{tr} F_{\alpha}^{c 3}\right)\right], \\
& X_{6}^{\prime(\alpha)}=\frac{-1}{6 \sqrt{6}(2 \pi)^{3}}\left[\frac{1}{16} \operatorname{tr} R^{2}\left(\operatorname{tr} F_{\alpha}^{a}+\operatorname{tr} F_{\alpha}^{b}-2 \operatorname{tr} F_{\alpha}^{c}\right)-\left(\operatorname{tr} F_{\alpha}^{a 3}+\operatorname{tr} F_{\alpha}^{b^{3}}-2 \operatorname{tr} F_{\alpha}^{c 3}\right)\right], \\
& X_{6}^{\prime \prime(\alpha)}=\frac{-1}{3 \sqrt{6}(2 \pi)^{3}}\left[\frac{1}{16} \operatorname{tr} R^{2}\left(\operatorname{tr} F_{\alpha}^{a}-\operatorname{tr} F_{\alpha}^{b}\right)-\left(\operatorname{tr} F_{\alpha}^{a 3}-\operatorname{tr} F_{\alpha}^{b^{3}}\right)\right], \\
& X_{2}^{(\alpha)}=\frac{-2}{\sqrt{3}(2 \pi)}\left(\operatorname{tr} F_{\alpha}^{a}+\operatorname{tr} F_{\alpha}^{b}+\operatorname{tr} F_{\alpha}^{c}\right), \\
& X_{2}^{\prime(\alpha)}=\frac{-1}{\sqrt{6}(2 \pi)}\left(\operatorname{tr} F_{\alpha}^{a}+\operatorname{tr} F_{\alpha}^{b}-2 \operatorname{tr} F_{\alpha}^{c}\right), \\
& X_{2}^{\prime \prime(\alpha)}=\frac{-3}{\sqrt{6}(2 \pi)}\left(\operatorname{tr} F_{\alpha}^{a}-\operatorname{tr} F_{\alpha}^{b}\right) .
\end{aligned}
$$

The corresponding inflow is

$$
\begin{aligned}
I= & X_{4}^{(5)} X_{4}^{(9)}-\left(X_{4}^{\prime(5)}-X_{4}^{\prime(9)}\right)\left(X_{4}^{\prime(5)}-X_{4}^{\prime(9)}\right)-\left(X_{6}^{\prime(5)}+X_{6}^{\prime(9)}\right)\left(X_{2}^{\prime(5)}+X_{2}^{\prime(9)}\right) \\
& -X_{4}^{\prime \prime(5)}\left(X_{4}^{\prime \prime(5)}-\frac{1}{3} X_{4}^{\prime \prime(9)}\right)-X_{4}^{\prime \prime(9)}\left(X_{4}^{\prime \prime(9)}-\frac{1}{3} X_{4}^{\prime \prime(5)}\right) \\
& -X_{6}^{\prime \prime(5)}\left(X_{2}^{\prime \prime(5)}+\frac{1}{3} X_{2}^{\prime \prime(9)}\right)-X_{6}^{\prime \prime(9)}\left(X_{2}^{\prime \prime(9)}+\frac{1}{3} X_{2}^{\prime \prime(5)}\right) \\
& -X_{6}^{(5)}\left(X_{2}^{(5)}+\frac{1}{4} X_{2}^{(9)}\right)-X_{6}^{(9)}\left(X_{2}^{(9)}+\frac{1}{4} X_{2}^{(5)}\right)
\end{aligned}
$$

and is equal and opposite to the total anomaly polynomial. 
Again, the form of the $U(1)$ combinations coupled to the various scalars can be checked through a disk computation, which in the $k$-twisted sector is again proportional to $\operatorname{tr}\left(\lambda \gamma_{k}\right)$. The Chan-Paton wave functions for the three $U(1)$ combinations $F_{\alpha}^{a}+F_{\alpha}^{b}-2 F_{\alpha}^{c}, F_{\alpha}^{a}-F_{\alpha}^{b}$ and $F_{\alpha}^{a}+F_{\alpha}^{b}+F_{\alpha}^{c}$ are $\lambda^{1}=\operatorname{diag}\left(\mathbf{I}_{\mathbf{4}},-\mathbf{I}_{\mathbf{4}}, \mathbf{I}_{\mathbf{4}},-\mathbf{I}_{\mathbf{4}},-\mathbf{I}_{\mathbf{8}}, \mathbf{I}_{\mathbf{8}}\right)$, $\lambda^{2}=\operatorname{diag}\left(\mathbf{I}_{\mathbf{4}},-\mathbf{I}_{\mathbf{4}},-\mathbf{I}_{\mathbf{4}}, \mathbf{I}_{\mathbf{4}}, 0 \mathbf{I}_{\mathbf{8}}, 0 \mathbf{I}_{\mathbf{8}}\right)$ and $\lambda^{3}=\operatorname{diag}\left(\mathbf{I}_{\mathbf{4}},-\mathbf{I}_{\mathbf{4}}, \mathbf{I}_{\mathbf{4}},-\mathbf{I}_{\mathbf{4}}, 1 / 2 \mathbf{I}_{\mathbf{8}},-1 / 2 \mathbf{I}_{\mathbf{8}}\right)$. Then, one finds as before $\operatorname{tr}\left(\lambda^{i} \gamma_{k}\right) \sim \delta_{k}^{i}$, again in agreement with the fact that the inflows due to the $k=1, k=2$ and $k=3$ twisted scalars do not mix.

\section{Field theory analysis}

In this section, we analyze some aspects of the low-energy effective action of the orientifold models discussed here. In particular, we focus on some interesting implications of the anomaly cancelling couplings found in section five and other terms related to them by $N=1 D=6$ supersymmetry.

\subsection{Anomalous $U(1)$ 's}

As already shown in detail for the $\mathbf{Z}_{2}$ case in [26], a generalized Green-Schwarz mechanism involving scalars induces an Higgs mechanism breaking various $U(1)$ factors of the gauge group. As expected, this feature is common to all IIB orientifold models discussed here. Differently from the $\mathbf{Z}_{2}$ case, however, one has in general to carefully establish whether the RR twisted scalars belong to hyper or tensor multiplets. Luckily, the answer can be derived from [31], where it was shown that the scalars belonging to the extra tensor multiplets are actually twisted NSNS scalars巴. This implies that all the RR twisted scalars involved in the inflow mechanism analyzed in section five belong to neutral hypermultiplets, like in the $\mathbf{Z}_{2}$ case. The analysis is then identical to that of [26]. After an integration by parts and a duality transformation, the anomalous couplings to the various four-forms reported in last section enter as corrections to the kinetic terms of the corresponding dual scalars. For these fields strength to be gauge invariant, the scalars have then to transform anomalously under the corresponding $U(1)$ gauge transformation. In order to write explicit expression, it is convenient to use the linear combinations of scalar fields entering the inflows as new independent fields $\lambda^{I}$. By doing so, the combinations $A^{I}$ of $U(1)$ gauge fields entering as shifts in the field-strengths

$$
H^{I}=d \lambda^{I}-\frac{A^{I}}{2 \pi}
$$

are directly related to the combinations $X_{2}^{I}$ of 2-forms in the corresponding factor of the inflows derived in section five, that is (5.1), (5.3), (5.7) and (5.11). The precise relation is $X_{2}^{I}=-1 / \pi d A^{I}$, so that the gauge field $A^{I}$ is essentially the first descent of $X_{2}: A^{I}=-\pi X_{2}^{I(0)}$. Also the anomalous transformations are easily deduced: under the gauge transformation $\delta A^{I}=d \epsilon^{I}$, the scalars transform as $\delta \lambda^{I}=\epsilon^{I} / 2 \pi$.

\footnotetext{
${ }^{11}$ We thank A. Sagnotti for very useful discussions about this point.
} 
Using the general results and the notation of section four, the anomalous interaction leading to the shift in the field strengths of the twisted scalars $\phi^{(k)}$ can be written in a very compact form. One finds

$$
S_{S C}^{(k)}=\sqrt{\frac{2}{\pi N}} \sin \frac{\pi k}{N} \int d^{6} x\left\{\partial_{\mu} \phi^{(k) 1} \operatorname{tr}\left[\gamma_{k} A_{5}^{\mu}\right]+\left(\frac{1}{\sqrt{N_{k}}} \sum_{i_{k}=1}^{N_{k}} \partial_{\mu} \phi^{(k) i_{k}}\right) \operatorname{tr}\left[\gamma_{k} A_{9}^{\mu}\right]\right\} .
$$

In the following, we report explicit expressions for each model in terms of the physical scalars

\section{$\mathrm{Z}_{2}$-model}

In the $\mathbf{Z}_{2}$ case, the combinations of scalars participating to the inflow and the corresponding $U(1)$ gauge fields are given by

$$
\begin{aligned}
& \lambda^{1}=\varphi^{1} \quad: A^{1}=A_{9}+\frac{1}{4} A_{5}, \\
& \lambda^{2}=\frac{1}{4} \sum_{j=1}^{16} \varphi^{j}: A^{2}=A_{5}+\frac{1}{4} A_{9} .
\end{aligned}
$$

The two scalars $\lambda^{1,2}$ are eaten by the two $U(1)$ gauge fields $A^{1,2}$ through a Higgs mechanism, and the latter become massive. Since supersymmetry remains unbroken, the whole hypermultiplets containing $\lambda^{1,2}$ must become massive. This is indeed what happens since the inhomogeneous $U(1)$ gauge transformations of the scalars $\lambda^{1,2}$ turn some D-terms into mass terms for the remaining scalars in the hypermultiplet [41, 26] and, most likely, the two Weyl fermions of opposite chiralities, belonging to the vector and hyper multiplet, combine into a single massive Dirac fermion. Since in this Higgsing the only states that disappear from the massless spectrum and contribute to gauge and gravitational anomalies are the two massless chiral fermions, of opposite chiralities, the model remains anomaly-free, with unbroken $(S U(16))^{2}$ gauge group.

\section{$\mathrm{Z}_{3}$-model}

In the $\mathbf{Z}_{3}$ case, there is only one combination of scalars and one $U(1)$ gauge field, given by

$$
\lambda=\frac{1}{3} \sum_{m=1}^{9} \phi^{\prime m}: A=\frac{\sqrt{3}}{2} A_{9}^{a} .
$$

By the same mechanism as before, the unbroken gauge group is found to be $S O(16) \times$ $S U(8)$.

\section{$\mathrm{Z}_{4}-$ model}

The $\mathbf{Z}_{4}$ model presents four different combinations of scalars and $U(1)$ gauge fields,

\footnotetext{
${ }^{12}$ Recall that some of the $\phi^{(k)}$ 's are identified. See Appendix B.
} 
given by

$$
\begin{aligned}
\lambda^{1} & =\phi^{\prime 1} & : A^{1} & =\frac{1}{2}\left[\left(A_{5}^{a}-A_{5}^{b}\right)+\frac{1}{2}\left(A_{9}^{a}-A_{9}^{b}\right)\right], \\
\lambda^{2} & =\frac{1}{2} \sum_{m=1}^{4} \phi^{\prime m} & : A^{2} & =\frac{1}{2}\left[\left(A_{9}^{a}-A_{9}^{b}\right)+\frac{1}{2}\left(A_{5}^{a}-A_{5}^{b}\right)\right], \\
\lambda^{3} & =\varphi^{1} & : A^{3} & =\frac{1}{\sqrt{2}}\left[\left(A_{5}^{a}+A_{5}^{b}\right)+\frac{1}{4}\left(A_{9}^{a}+A_{9}^{b}\right)\right], \\
\lambda^{4} & =\frac{1}{4}\left(\sum_{j=1}^{4} \varphi^{j}+\sqrt{2} \sum_{j=5}^{10} \varphi^{j}\right) & : A^{4} & =\frac{1}{\sqrt{2}}\left[\left(A_{9}^{a}+A_{9}^{b}\right)+\frac{1}{4}\left(A_{5}^{a}+A_{5}^{b}\right)\right] .
\end{aligned}
$$

All the four $U(1)$ factors are then spontaneously broken, leaving as unbroken gauge group $(S U(8) \times S U(8))^{2}$.

\section{$\mathrm{Z}_{6}$-model}

The $\mathbf{Z}_{6}$ model presents the new feature that a $U(1)$ factor is left unbroken. Indeed, there are only five independent combinations of scalars involved in the Higgs mechanism, corresponding to five combinations of the six $U(1)$ fields:

$$
\begin{aligned}
\lambda^{1} & =\phi^{\prime} & : A^{1} & =\frac{1}{2 \sqrt{6}}\left[\left(A_{5}^{a}+A_{5}^{b}-2 A_{5}^{c}\right)+\left(A_{9}^{a}+A_{9}^{b}-2 A_{9}^{c}\right)\right], \\
\lambda^{2} & =\phi^{\prime \prime 1} & : A^{2} & =\frac{3}{2 \sqrt{6}}\left[\left(A_{5}^{a}-A_{5}^{b}\right)+\frac{1}{3}\left(A_{9}^{a}-A_{9}^{b}\right)\right], \\
\lambda^{3} & =\frac{1}{3}\left(\phi^{\prime \prime 1}+\sqrt{2} \sum_{m=2}^{5} \phi^{\prime m}\right) & : A^{3} & =\frac{3}{2 \sqrt{6}}\left[\left(A_{9}^{a}-A_{9}^{b}\right)+\frac{1}{3}\left(A_{5}^{a}-A_{5}^{b}\right)\right], \\
\lambda^{4} & =\varphi^{1} & : A^{4} & =\frac{1}{\sqrt{3}}\left[\left(A_{5}^{a}+A_{5}^{b}+A_{5}^{c}\right)+\frac{1}{4}\left(A_{9}^{a}+A_{9}^{b}+A_{5}^{c}\right)\right], \\
\lambda^{5} & =\frac{1}{4}\left(\sum_{j=1}^{4} \varphi^{j}+\sqrt{3} \sum_{j=2}^{6} \varphi^{j}\right) & : A^{5} & =\frac{1}{\sqrt{3}}\left[\left(A_{9}^{a}+A_{9}^{b}+A_{5}^{c}\right)+\frac{1}{4}\left(A_{5}^{a}+A_{5}^{b}+A_{5}^{c}\right)\right] .
\end{aligned}
$$

The resulting unbroken gauge group is $(S U(4) \times S U(4) \times S U(8))^{2} \times U(1)$. It is straightforward to determine how the surviving $U(1)$ factor is embedded in the original gauge group. Indeed, the corresponding gauge field is the linear combination which is orthogonal to $A^{1-5}$, and is found to be similar to $A^{1}$, but with a relative minus sign between 5 and 9 factors.

A similar analysis can be performed in the more general case where we have arbitrary configurations for the D5 branes and non-vanishing Wilson lines in the nine branes gauge groups. Although we do not report this more general analysis, it is clear that in any configuration the maximum number of $U(1)$ 's that can be Higgsed cannot exceed the number of RR twisted closed string scalars present in each model.

\subsection{Gauge couplings}

Let us now turn our attention to the Chern-Simons couplings of the tensor fields. The most remarkable property of these terms is that their structure is related by supersymmetry to the gauge couplings constants $1 / g^{2}$ of the theory [25]. Whenever some of them vanish or become negative, tensionless strings appear and the model 
undergoes a phase transition [28, 29, 30]. It is then extremely useful to analyze in detail anomaly cancellation, being a useful tool to address such questions.

In order to be more concrete, we briefly review here some general properties of $N=1 D=6$ supergravity coupled to $n_{T}$ tensor multiplets and vector multiplets of some gauge group. For details we refer the reader to [42 and references therein. The coupling to the hypermultiplet sector does not play any role in the discussion that will follow and it will be omitted[ᄑ]. The kinetic terms for the bosonic fields of the theory, including the Chern-Simons couplings for the two-forms, are 42

$$
\begin{aligned}
e^{-1} \mathcal{L}_{\text {bos. }}^{\text {kin. }}= & -\frac{1}{4} R+\frac{1}{12} G_{r s} H_{\mu \nu \rho}^{r} H^{s, \mu \nu \rho}-\frac{1}{4}\left(\partial_{\mu} v^{r}\right)\left(\partial^{\mu} v_{r}\right) \\
& -\frac{1}{2} v_{r} c_{z}^{r} \operatorname{tr}_{z} F_{\mu \nu} F^{\mu \nu}-6 e^{-1} c_{r z} b^{r} \wedge \operatorname{tr}_{z} F^{2} .
\end{aligned}
$$

The physical scalars $v_{r}, r=0, \ldots, n_{T}$, parametrize the coset space $S O\left(1, n_{T}\right) / S O\left(n_{T}\right)$, whereas the non-propagating scalars $x_{r}^{M}, M=1, \ldots, n_{T}$, can be gauged away by fixing the local $S O\left(n_{T}\right)$ symmetry; together, $v_{r}$ and $x_{r}^{M}$ form an $S O\left(1, n_{T}\right)$ matrix. Moreover, $e$ is the determinant of the sechsbein, and $G_{r s}=v_{r} v_{s}+x_{r}^{M} x_{s}^{M}$. Finally, $H^{r}=d b^{r}-c_{z}^{r} w_{3}^{z}$ are the modified field-strengths of the tensors, shifted by the ChernSimons three-forms $w_{3}^{z}$ defined as descents of $\operatorname{tr}_{z} F^{2}: d w_{3}^{z}=\operatorname{tr}_{z} F^{2}$; the $c_{z}^{r}$ are constants, and $z$ labels different factors of the gauge group. The crucial observation of 25] is that the same coefficients $c_{z}^{r}$ appear in the Bianchi identities and couplings for $b^{r}$ and in the gauge couplings. Among the $n_{T}+1$ field strengths $H^{r}, H^{(+)}=v_{r} H^{r}$ is self-dual, while $K^{M(-)}=x_{r}^{M} H^{r}$ are anti-self-dual. The Lagrangian (6.2) presents a manifest global $S O\left(1, n_{T}\right)$ symmetry rotating the tensor fields, the scalars and the constants $c_{z}^{r}$. In writing a local covariant Lagrangian as in (6.2), it is understood that the (anti)self-duality constraints for the field strengths must be imposed only after varying the Lagrangian, at the level of equations of motion. Alternatively, following the work of [40], one could add an auxiliary scalar field and additional terms to the model that take into account the (anti)self-duality conditions. We will not do that, since this will be irrelevant for our considerations, but notice that such Lagrangian, including all four-fermion terms, already appeared in 44].

In order to better understand how to apply these general considerations to the IIB orientifold models analyzed here, it is useful to consider first a model with $n_{T}=1$ and arbitrary gauge group. In this case, the $S O(1,1)$ matrix is simply parametrized by $v_{0}=\cosh \phi=x_{1}^{1}, v_{1}=\sinh \phi=x_{0}^{1}$. We can also combine the self-dual and anti-self-dual tensors to form an unconstrained two-form field. Choosing the simple combination $b=1 / 2\left(b^{0}-b^{1}\right)$, the Lagrangian (6.2) becomes

$$
\begin{aligned}
e^{-1} \mathcal{L}_{\text {bos. }}^{\text {kin. }}= & -\frac{1}{4} R+\frac{1}{6} e^{-2 \phi} H_{\mu \nu \rho} H^{\mu \nu \rho}-\frac{1}{4}\left(\partial_{\mu} v^{r}\right)\left(\partial^{\mu} v_{r}\right) \\
& -\frac{1}{4}\left(e^{\phi} c_{z}+e^{-\phi} \tilde{c}_{z}\right) \operatorname{tr}_{z} F_{\mu \nu} F^{\mu \nu}-6 e^{-1} c_{z} b \wedge \operatorname{tr}_{z} F^{2},
\end{aligned}
$$

\footnotetext{
${ }^{13}$ See 43 for an analysis of the hypermultiplet sector.
} 
where $c_{z}=c_{z}^{0}+c_{z}^{1}, \tilde{c}_{z}=c_{z}^{0}-c_{z}^{1}$ and $H=d b-\tilde{c}_{z} / 2 w_{3}^{z}$. We dropped from (6.3) the other linear combination $\tilde{b}=1 / 2\left(b^{0}+b^{1}\right)$ since its dynamics is completely determined from that of $b$. Let us connect this Lagrangian with the one expected from type I compactified on K3. In presence of D5-branes, the ten dimensional kinetic terms for $b$ and the $F_{9,5}$ field strengths are schematically, in the string frame:

$$
e_{S}^{-1} \mathcal{L}_{k i n .}^{(10)} \sim e^{-2 D_{10}} R_{S}+H^{2}+e^{-D_{10}} \operatorname{tr} F_{9}^{2}+e^{-D_{10}} \delta^{(4)}(y) \operatorname{tr} F_{5}^{2}
$$

with $D_{10}$ the ten dimensional dilaton. Compactifying on $D=6$ and going to the Einstein frame, one gets

$$
e_{E}^{-1} \mathcal{L}_{\text {kin. }}^{(6)} \sim R_{E}+V_{4} H^{2}+V_{4}^{1 / 2} \operatorname{tr} F_{9}^{2}+V_{4}^{-1 / 2} \operatorname{tr} F_{5}^{2},
$$

where $V_{4}$ is the volume of $K 3$. By comparing (6.5) with (6.3), we see that $V_{4}=e^{-2\langle\phi\rangle}$, $\phi$ being the scalar in the tensor multiplet. Moreover the comparison requires also that $c_{9}=\tilde{c}_{5}=0$. This is the case for the $\mathbf{Z}_{2}$ orientifold model, where these considerations apply. We found in last section that the tensor field $b$ couples only to the gauge group of the 5 sector through $X_{4}^{(5)}$, whereas its Bianchi identity, obtained by integrating by parts and dualizing the coupling to $\tilde{b}$, involves only the 9 sector. Moreover $c_{5}=\tilde{c}_{9}>0$; no phase transitions can occur in this case.

Let us consider now the other orientifold models with $n_{T}>1$. The first task is to identify the combination of anti-self-dual and self-dual tensor fields giving rise to the unconstrained untwisted $b$ field and to the remaining $n_{T}-1$ tensors. Although these models do not have a clear geometric interpretation, the kinetic term for the untwisted tensor field $b$, being independent of the details of the orientifold, can still be seen as a reduction from $D=10$ to $D=6$ and has to be multiplied just by the volume $V_{4}$ of the internal orbifold. In this case, we define the scalar $\phi$ such that $V_{4}=e^{-2\langle\phi\rangle}$. Let us now parametrize the scalar fields $v_{r}$ as $v_{0}=\cosh \phi, v_{i}=$ $\Omega_{i} \sinh \phi, i=1, \ldots, n_{T}$, where $\Omega_{i}$ are coordinates on the unit $S^{\left(n_{T}-1\right)}$-sphere $\left(\sum_{i} \Omega_{i}^{2}=\right.$ $1)$. With this choice of parametrization, there is a natural (and probably unique) choice for the vacuum expectation values for the scalars $v_{r}$ and $x_{r}^{M}$, that reproduce the kinetic term for $H:\langle v\rangle_{0}=\cosh \langle\phi\rangle=\langle x\rangle_{1}^{1},\langle v\rangle_{1}=\sinh \langle\phi\rangle=\langle x\rangle_{0}^{1},\langle x\rangle_{i}^{I}=\delta_{i}^{I}$ for $i, I=2, \ldots, n_{T}$, all the other being equal to zero. This strongly suggests that the orientifold string construction is appropriate only at this point in the moduli space parametrized by the vacuum expectation values of the scalars belonging to the extra tensor multiplets. Notice that the vacuum $\left\langle\Omega_{i}\right\rangle=0$ breaks the global $S O\left(1, n_{T}\right)$ symmetry spontaneously to $S O(1,1) \times S O\left(n_{T}-1\right)$. The untwisted $b$ field is obtained precisely like in the $n_{T}=1$ case whereas $b^{i}=K^{i(-)}$ are identified with the additional $n_{T}-1$ anti-self-dual two-forms. Correspondingly, at this point in moduli space the gauge coupling constants depend only on the coefficients $c_{z}$ and $\tilde{c}_{z}$ associated to the Bianchi identity and couplings of $b$ only. Notice that for all the $\mathbf{Z}_{N}$ models $c_{9}=\tilde{c}_{5}=0$ and $c_{5}=\tilde{c}_{9}>0$. Again, all the gauge kinetic terms are positive definite at this particular point in the moduli space. 
All the coefficients $c_{r}^{z}$ do not depend on the vev's of the scalars $v_{r}$ and can be fixed from the four-forms $X_{4}$ found in last section. This allows to analyze the gauge couplings for generic values of the moduli. We have checked that for each of the models considered here there exist a continuous family of points in moduli space where the gauge couplings vanish and tensionless strings occur. A detailed analysis of the loci where this phenomenon occurs and their interpretation would be extremely interesting. As an illustrative example, consider the $\mathbf{Z}_{3}$ model. Modulo an irrelevant overall factor, the constants $c_{z}^{r}$ for the two factors $z=a, b$ of the gauge group are found to be $c_{a}^{0}=-c_{a}^{1}=1 / 2 \sqrt{6}, c_{b}^{0}=-c_{b}^{1}=1 / 4 \sqrt{6}, c_{a}^{m}=1 / 6 \sqrt{2}, c_{b}^{m}=-1 / 6 \sqrt{2}$, $m=1, \ldots, 9$. It is then easy to check that for generic values of $\left\langle\Omega_{i}\right\rangle$, there exists in general a value of $\langle\phi\rangle$, corresponding to a particular $V_{4}$, for which $v_{r} c_{z}^{r}$ vanishes. Choosing for instance $\left\langle\Omega_{1}\right\rangle=0,\left\langle\Omega_{i>1}\right\rangle=1 / 3$, the gauge couplings of $F_{a}$ and $F_{b}$ vanish for $\operatorname{coth}\langle\phi\rangle=\sqrt{3}$ and $\operatorname{coth}\langle\phi\rangle=2 \sqrt{3}$ respectively.

The linear couplings of the scalars to the field strengths can also be derived once the constants $c_{z}^{r}$ are known. Using the general results and notation of section four, these couplings can be rewritten in a concise way as

$$
S_{G C}^{(k)}=\frac{1}{32 \pi^{2}} \sqrt{\frac{\pi}{N}} \sin \frac{\pi k}{N} \int d^{6} x\left\{\chi^{(k) 1} \operatorname{tr}\left[\gamma_{k} F_{5}^{2}\right]+\left(\frac{1}{\sqrt{N_{k}}} \sum_{i_{k}=1}^{N_{k}} \chi^{(k) i_{k}}\right) \operatorname{tr}\left[\gamma_{k} F_{9}^{2}\right]\right\},
$$

where $\chi^{(k)}, k=1, \ldots, N / 2-1$ are the NSNS twisted scalars belonging to the additional tensor multiplets, and $F_{5,9}$ are now conventional field-strengths rather than curvature two-forms. As for the 9 sector, the above couplings are in agreement with the recent results of [31, modulo an overall numerical coefficient that we have not attempted to check ${ }^{\text {th }}$.

\section{Discussion and conclusions}

In this paper, we have computed particular topological amplitudes in the odd spinstructure enabling us to understand in detail how anomalies cancel in a class of $N=1$ $D=6$ orientifold models with maximal unbroken gauge group. By factorization, we have also found the D-brane, O-plane and fixed-point couplings to the RR fields arising in these models.

The mechanism of anomaly cancellation applies in its most general form, with the exchange of different tensor fields [25] and, whenever Abelian factors are present, scalar fields as well [11, 26]. The RR scalars involved in the inflow mechanism belong always to neutral hypermultiplets and are responsible for a spontaneous symmetry breaking of all $U(1)$ factors but one in the $\mathbf{Z}_{6}$ model, in the maximally symmetric case. More generically, the maximum number of $U(1)$ that can be spontaneously broken never exceeds the number of neutral hypermultiplets arising from the twisted closed string sector.

\footnotetext{
${ }^{14}$ The scalar fields $m_{k}$ of [3] have to be identified as $m_{k}=1 / \sqrt{N_{k}} \sum_{i_{k}=1}^{N_{k}} \chi^{(k) i_{k}}$.
} 
By comparing some terms in the low-energy effective actions of these models with the most general ones allowed by supersymmetry, we deduce that the vacuum expectation values of the scalars belonging to the extra tensor multiplets are fixed at particular values. In these points in moduli space all the gauge kinetic terms are definite positive. However, varying these moduli - flat directions in the low-energy action - we always find special loci where the gauge couplings diverge and tensionless strings occur. Analogously, we can also fix the gauge couplings of these scalars for both the D9 and D5 gauge groups.

The models we discussed in this paper are particular examples of six-dimensional theories with extra tensor multiplets. These arise for instance in F and M-theory $D=6$ compactifications ${ }^{\text {to }}$. In this last case, each extra tensor multiplet is believed to come from an M5-brane in the bulk of the $11^{\text {th }}$ dimension [46]. Although the nonperturbative heterotic Spin(32)/ $\mathbf{Z}_{2}$ duals to the IIB orientifolds models are known [47, their explicit realization as M-theory vacua is not a simple issue, especially in the maximally symmetric case discussed here. Nevertheless, some questions might still be qualitatively addressed. Assuming that each extra tensor multiplet comes from a single M5-brane, one might naively hope that these models are somehow related to Mtheory compactified on an orbifold like $T^{4} / \mathbf{Z}_{N} \times S^{1} / \mathbf{Z}_{2}$. However, from 48] it is clear that such M-theory vacua corresponds to non-perturbative vacua of strongly coupled $E_{8} \times E_{8}$ heterotic theory on $T^{4} / \mathbf{Z}_{N}$, with total instanton number $25-n_{T}$. These vacua could in turn be related to $K 3$ orientifolds. The $n_{T}-1 \mathrm{M} 5$-branes are located at distinct fixed-points of the orbifold. One is also tempted to consider somehow the vev $\left\langle\Omega_{i}\right\rangle$ as the moduli corresponding to move the $i^{t h}$ brane on the $S^{1} / \mathbf{Z}_{2}$ segment. It seems that the orientifold description requires all these M5-branes to be stuck at some point in the segment and at different fixed-points on the $T^{4} / \mathbf{Z}_{N}$ orbifold. On the other hand, in M-theory one has the freedom of moving the M5-branes along the segment by turning on $\left\langle\Omega_{i}\right\rangle$. As already said, this leads to tensionless strings at special points. It would be then very interesting to better understand the connection with M-theory by a full and deeper analysis of this phenomenon. In this direction, a more general analysis with different configurations for the D5 branes and with Wilson lines turned on, would also be quite interesting.

Generalizing the idea of 48, in compactifying M-theory on orbifolds new twisted sectors are expected to arise at fixed-points. These will play a decisive role in making the theory anomaly-free. It would be then extremely interesting to see how new couplings to the twisted sectors, as well as the compactification of the $D=11$ ChernSimons term $C_{3} \wedge X_{8}$ on orbifolds, are related to the anomalous couplings found here. This could yield important informations on M-theory at the microscopic level.

\footnotetext{
${ }^{15}$ See also 45] for other constructions of these vacua.
} 


\section{Acknowledgments}

It is a pleasure to thank D. Ghoshal, A. Sagnotti and S. Theisen for enlightening discussions and very useful comments. We are also grateful to D. Bernardini, J. Pawelczyk and E. Scheidegger for interesting and continuous exchange of ideas. C.A.S. also thanks the University of Amsterdam for hospitality.

This work has been supported by EEC under TMR contract ERBFMRX-CT960045 and by the Nederlandse Organisatie voor Wetenschappelijk Onderzoek (NWO).

\section{A. Inflows}

In this appendix, we recall some formulae from [5] which are needed to check that the anomalous couplings that we have proposed indeed reproduce the inflows from which they have been derived.

Sector $\mathrm{k}=0$

Two anomalous coupling of the form

$$
S_{A}=\sqrt{2 \pi} \int_{A} C_{(0)} \wedge A(F, R), \quad S_{B}=\sqrt{2 \pi} \int_{B} \tilde{C}_{(0)} \wedge B(F, R)
$$

with $C_{(0)}, \tilde{C}_{(0)}$ given by $(4.4)$, produce an inflow on the $\mathrm{AB}$ intersection given by $\delta_{\eta}\left(i S_{A+B}\right)=2 \pi i \int I_{A B}^{(1)}$ where

$$
I_{A B}=\frac{1}{2}\left[A_{4}(F, R) B_{4}(-F, R)+A_{4}(-F, R) B_{4}(F, R)\right] .
$$

In this case all the couplings are even in $F$ due to the property $\operatorname{ch}(-F)=\operatorname{ch}(F)$ of the Chern class in the Chan-Paton representation. The inflow then reduces to

$$
I_{A B}=A_{4}(F, R) B_{4}(F, R)=[A(F, R) \wedge B(F, R)]_{8} .
$$

Of course we neglect here and in the following the eight-form components $A_{8}(F, R)$ and $B_{8}(F, R)$, because their sum automatically vanishes once the tadpole conditions are solved, and therefore there is no inflow associated with them. For non-distinct objects, an additional $1 / 2$ is needed.

\section{Sector $\mathrm{k}=\mathrm{N} / 2$}

Two anomalous coupling of the form

$$
S_{A}=\sqrt{2 \pi} \int_{A} C_{(N / 2)}^{i} \wedge A(F, R), \quad S_{B}=\sqrt{2 \pi} \int_{B} C_{(N / 2)}^{i} \wedge B(F, R)
$$

with $C_{(N / 2)}^{i}$ given by (4.11), produce an inflow on the $\mathrm{AB}$ intersection given by

$$
\begin{aligned}
I_{A B}=\frac{1}{2}[ & A_{2}(F, R) B_{6}(-F, R)+A_{6}(F, R) B_{2}(-F, R) \\
& \left.\quad+A_{2}(-F, R) B_{6}(F, R)+A_{6}(-F, R) B_{2}(F, R)\right] .
\end{aligned}
$$


Now all the couplings are odd in $F$ due to the property $\operatorname{ch}\left(\gamma_{N / 2}(-F)\right)=-\operatorname{ch}\left(\gamma_{N / 2} F\right)$ in the Chan-Paton representation. The inflow then reduces to

$$
I_{A B}=-A_{2}(F, R) B_{6}(F, R)-A_{6}(F, R) B_{2}(F, R)=-[A(F, R) \wedge B(F, R)]_{8} .
$$

For non-distinct objects, an additional $1 / 2$ is needed.

\section{Sectors $k \neq 0, N / 2$}

The anomalous couplings

$$
S_{A}=\sqrt{2 \pi} \int_{A} C_{(k)}^{i_{k}} \wedge A(F, R), \quad S_{B}=\sqrt{2 \pi} \int_{B} C_{(k)}^{i_{k}} \wedge B(F, R)
$$

with $C_{(k)}^{i_{k}}$ as in (4.16), produce an inflow given by

$$
\begin{aligned}
I_{A B}=\frac{1}{2}[ & A_{2}(F, R) B_{6}(-F, R)+A_{6}(F, R) B_{2}(-F, R) \\
& +A_{2}(-F, R) B_{6}(F, R)+A_{6}(-F, R) B_{2}(F, R) \\
& \left.-A_{4}(-F, R) B_{4}(F, R)-A_{4}(-F, R) B_{4}(F, R)\right] .
\end{aligned}
$$

Furthermore, the 4 -form couplings are even in $F$ whereas the 2 and 6 -form couplings are odd, due to the property $\left.\operatorname{ch}\left(\gamma_{k}(-F)\right)\right|_{p}=\left.(-)^{p / 2} \operatorname{ch}\left(\gamma_{k} F\right)\right|_{p}$ valid for the p-form component. The inflow then reduces to

$$
\begin{aligned}
I_{A B} & =-A_{2}(F, R) B_{6}(F, R)-A_{6}(F, R) B_{2}(F, R)-A_{4}(F, R) B_{4}(F, R) \\
& =-[A(F, R) \wedge B(F, R)]_{8} .
\end{aligned}
$$

For non-distinct objects, an additional $1 / 2$ is needed as usual.

\section{B. Anomalous couplings}

We report here for completeness the Wess-Zumino couplings computed in section five, but in a form that makes clearer the different contribution due to D9-branes, D5-branes, O9-planes, O5-planes and Fk fixed-points.

\section{$\mathbf{Z}_{2}$-model}

There are 32 D9-branes, 32 D5-branes, 1 O9-plane and 16 O5-planes or F1 fixed-points ( $\mathbf{Z}_{2}$-fixed). Applying the general results of section four, their anomalous couplings are found to be

$$
\begin{aligned}
& S_{D 5}=\sqrt{2 \pi} \int\left\{\frac{1}{2} b \operatorname{ch}\left(F_{5}\right)-(\varphi+\tilde{\varphi})^{1} \operatorname{ch}\left(\gamma_{1} F_{5}\right)\right\} \sqrt{\hat{A}(R)}, \\
& S_{D 9}=\sqrt{2 \pi} \int\left\{\frac{1}{2} \tilde{b} \operatorname{ch}\left(F_{9}\right)+\frac{1}{4} \sum_{j=1}^{16}(\varphi+\tilde{\varphi})^{j} \operatorname{ch}\left(\gamma_{2} F_{9}\right)\right\} \sqrt{\hat{A}(R)}, \\
& S_{O 5}=-\sqrt{2 \pi} \int b \sqrt{\hat{L}(R)}, \\
& S_{O 9}=-\sqrt{2 \pi} \int \tilde{b} \sqrt{\hat{L}(R)} .
\end{aligned}
$$


It is understood that all the products are wedge products and that one has to pick up the six-form component of each term only.

\section{$\mathrm{Z}_{3}$-model}

There are 32 D9-branes, 1 O9-plane and 9 F1 fixed-points $\left(\mathbf{Z}_{3}\right.$-fixed), with anomalous couplings given by

$$
\begin{aligned}
& S_{D 9}=\sqrt{2 \pi} \int\left\{\frac{1}{6} \tilde{b} \operatorname{ch}\left(F_{9}\right)+\frac{1}{3 \sqrt{2}} \sum_{m=1}^{9}\left[b^{\prime}-\tilde{b}^{\prime}+\sqrt{2}\left(\phi^{\prime}+\tilde{\phi}^{\prime}\right)\right]^{m} \operatorname{ch}\left(\gamma_{1} F_{9}\right)\right\} \sqrt{\hat{A}(R)} \\
& S_{O 9}=-\sqrt{2 \pi} \int \frac{2}{\sqrt{6}} \tilde{b} \sqrt{\hat{L}(R)} \\
& S_{F_{1}}=-\sqrt{2 \pi} \int \frac{1}{6 \sqrt{2}} \sum_{m=1}^{9}\left(b^{\prime}-\tilde{b}^{\prime}\right)^{m} \sqrt{\hat{L}(R)}
\end{aligned}
$$

\section{$\mathrm{Z}_{4}$-model}

There are 32 D9-branes, 32 D5-branes, 1 O9-plane, 16 O5-planes or F2-fixed-points $\left(\mathbf{Z}_{2}\right.$-fixed), and 4 F1-fixed-points $\left(\mathbf{Z}_{4}\right.$-fixed). Among the 16 scalars $\varphi_{(N / 2)}^{i}$ of the $\mathbf{Z}_{2}$-twisted sector, only $10, \varphi^{j}$, are physical. These are defined as $\varphi^{j}=\varphi_{(N / 2)}^{j}$ for $j=1, \ldots, 4$, and $\varphi^{j}=\sqrt{2}\left(\varphi_{(N / 2)}^{j}+\varphi_{(N / 2)}^{j+6}\right)$ for $j=5, \ldots, 10$. The anomalous couplings are then found to be

$$
\begin{aligned}
& S_{D 5}=\sqrt{2 \pi} \int\left\{\frac{1}{2 \sqrt{2}} b \operatorname{ch}\left(F_{5}\right)-\frac{1}{2}\left[b^{\prime}-\tilde{b}^{\prime}+\sqrt{2}\left(\phi^{\prime}+\tilde{\phi}^{\prime}\right)\right]^{1} \operatorname{ch}\left(\gamma_{1} F_{5}\right)\right. \\
& \left.-\frac{1}{\sqrt{2}}(\varphi+\tilde{\varphi})^{1} \operatorname{ch}\left(\gamma_{2} F_{5}\right)\right\} \sqrt{\hat{A}(R)}, \\
& S_{D 9}=\sqrt{2 \pi} \int\left\{\frac{1}{2 \sqrt{2}} \tilde{b} \operatorname{ch}\left(F_{9}\right)+\frac{1}{4} \sum_{m=1}^{4}\left[b^{\prime}-\tilde{b}^{\prime}+\sqrt{2}\left(\phi^{\prime}+\tilde{\phi}^{\prime}\right)\right]^{m} \operatorname{ch}\left(\gamma_{1} F_{9}\right)\right. \\
& \left.+\frac{1}{4 \sqrt{2}}\left[\sum_{j=1}^{4}(\varphi+\tilde{\varphi})^{j}+\sqrt{2} \sum_{j=5}^{10}(\varphi+\tilde{\varphi})^{j}\right] \operatorname{ch}\left(\gamma_{2} F_{9}\right)\right\} \sqrt{\hat{A}(R)}, \\
& S_{O 5}=-\sqrt{2 \pi} \int \frac{1}{\sqrt{2}} b \sqrt{\hat{L}(R)}, \\
& S_{O 9}=-\sqrt{2 \pi} \int \frac{1}{\sqrt{2}} \tilde{b} \sqrt{\hat{L}(R)}, \\
& S_{F 1}=0 \text {. }
\end{aligned}
$$

\section{$\mathrm{Z}_{6}$-model}

There are 32 D9-branes, 32 D5-branes, 1 O9-plane, 16 O5-planes or F3 fixed-points $\left(\mathbf{Z}_{2}\right.$-fixed), 1 F1 fixed-point $\left(\mathbf{Z}_{6}\right.$-fixed), and $9 \mathrm{~F} 2$ fixed-points $\left(\mathbf{Z}_{6}\right.$-fixed). Among the 16 scalars $\varphi_{(N / 2)}^{i}$ of the $\mathbf{Z}_{2}$-twisted sector, only $6, \varphi^{j}$, are physical. These are defined as $\varphi^{1}=\varphi_{(N / 2)}^{1}$ and $\varphi^{j}=\sqrt{3}\left(\varphi_{(N / 2)}^{j}+\varphi_{(N / 2)}^{j+5}+\varphi_{(N / 2)}^{j+10}\right)$ for $j=2, \ldots, 6$. Similarly, among the 9 copies of scalars and 2-forms $\phi_{(2)}^{i}, b_{(2) \mu \nu}^{i}$ of the $\mathbf{Z}_{3}$-twisted sector, only 5 
copies, $\phi^{\prime \prime m}, b_{\mu \nu}^{\prime \prime m}$, are independent. These are defined as $\phi^{\prime \prime 1}=\phi_{(N / 2)}^{1}, b_{\mu \nu}^{\prime \prime 1}=b_{(2) \mu \nu}^{1}$, and $\phi^{\prime \prime m}=\sqrt{2}\left(\phi_{(2)}^{m}+\varphi_{(2)}^{m+4}\right), b_{\mu \nu}^{\prime \prime m}=\sqrt{2}\left(b_{(2) \mu \nu}^{m}+b_{(2) \mu \nu}^{m+4}\right)$ for $m=2, \ldots, 5$. The anomalous couplings are then found to be

$$
\begin{aligned}
& S_{D 5}=\sqrt{2 \pi} \int\left\{\frac{1}{2 \sqrt{3}} b \operatorname{ch}\left(F_{5}\right)-\frac{1}{2 \sqrt{3}}\left[b^{\prime}-\tilde{b}^{\prime}+\sqrt{2}\left(\phi^{\prime}+\tilde{\phi}^{\prime}\right)\right] \operatorname{ch}\left(\gamma_{1} F_{5}\right)\right. \\
& -\frac{1}{2}\left[b^{\prime \prime}-\tilde{b}^{\prime \prime}+\sqrt{2}\left(\phi^{\prime \prime}+\tilde{\phi}^{\prime \prime}\right)\right]^{1} \operatorname{ch}\left(\gamma_{2} F_{5}\right) \\
& \left.-\frac{1}{\sqrt{3}}(\varphi+\tilde{\varphi})^{1} \operatorname{ch}\left(\gamma_{3} F_{5}\right)\right\} \sqrt{\hat{A}(R)} \\
& S_{D 9}=\sqrt{2 \pi} \int\left\{\frac{1}{2 \sqrt{3}} \tilde{b} \operatorname{ch}\left(F_{9}\right)+\frac{1}{2 \sqrt{3}}\left[b^{\prime}-\tilde{b}^{\prime}+\sqrt{2}\left(\phi^{\prime}+\tilde{\phi}^{\prime}\right)\right] \operatorname{ch}\left(\gamma_{1} F_{9}\right)\right. \\
& +\frac{1}{6}\left[\left[b^{\prime \prime}-\tilde{b}^{\prime \prime}+\sqrt{2}\left(\phi^{\prime \prime}+\tilde{\phi}^{\prime \prime}\right)\right]^{1}+\sqrt{2} \sum_{m=2}^{5}\left[b^{\prime \prime}-\tilde{b}^{\prime \prime}+\sqrt{2}\left(\phi^{\prime \prime}+\tilde{\phi}^{\prime \prime}\right)\right]^{m}\right] \operatorname{ch}\left(\gamma_{2} F_{9}\right) \\
& \left.+\frac{1}{4 \sqrt{3}}\left[(\varphi+\tilde{\varphi})^{1}+\sqrt{3} \sum_{j=2}^{6}(\varphi+\tilde{\varphi})^{j}\right] \operatorname{ch}\left(\gamma_{3} F_{9}\right)\right\} \sqrt{\hat{A}(R)}, \\
& S_{O 5}=-\sqrt{2 \pi} \int \frac{1}{\sqrt{3}} b \sqrt{\hat{L}(R)} \text {, } \\
& S_{O 9}=-\sqrt{2 \pi} \int \frac{1}{\sqrt{3}} \tilde{b} \sqrt{\hat{L}(R)} \\
& S_{F 1}=-\sqrt{2 \pi} \int \frac{1}{4}\left(b^{\prime \prime}-\tilde{b}^{\prime \prime}\right)^{1} \sqrt{\hat{L}(R)} \text {, } \\
& S_{F 2}=-\sqrt{2 \pi} \int-\frac{1}{12}\left[\left(b^{\prime \prime}-\tilde{b}^{\prime \prime}\right)^{1}+\sqrt{2} \sum_{m=2}^{5}\left(b^{\prime \prime}-\tilde{b}^{\prime \prime}\right)^{m}\right] \sqrt{\hat{L}(R)} .
\end{aligned}
$$

\section{References}

[1] M.B. Green and J.H. Schwarz, Anomaly cancellations in supersymmetric $D=10$ gauge theory and superstring theory, Phys. Lett. B149 (1984) 117-122; The hexagon gauge anomaly in type I superstring theory, Nucl. Phys. B255 (1985) 93114; Infinity cancellation in SO(32) superstring theory, Phys. Lett. B151 (1985) 21-25.

[2] C.G. Callan and J.A. Harvey, Anomalies and fermion zero modes on strings and domain walls, Nucl. Phys. B250 (1985) 427-436.

[3] J. Polchinski, Dirichlet-branes and Ramond-Ramond charges, Phys. Rev. Lett. 75 (1995) 4724-4727, hep-th/9510017.

[4] M.B. Green, J.A. Harvey and G. Moore, I-brane inflow and anomalous couplings on D-branes, Class. Quant. Grav. 14 (1997) 47-52, hep-th/9605033.

[5] Y-K.E. Cheung and Z. Yin, Anomalies, branes, and currents, Nucl. Phys. B517 (1998) 69-91, hep-th/9710206.

[6] C.A. Scrucca and M. Serone, Anomalies and inflow on D-branes and O-planes, Nucl. Phys. B556 (1999) 197-221, hep-th/9903145. 
[7] J.F. Morales, C.A. Scrucca and M. Serone, Anomalous couplings for D-branes and O-planes, Nucl. Phys. B552 (1999) 291-315, hep-th/9812071.

[8] M. Li, Boundary states of D-branes and Dy-strings, Nucl. Phys. B460 (1996) 351-361, hep-th/9510161.

[9] M.R. Douglas, Branes within branes, in Cargese 1997, strings, branes and dualities, ed. L. Baulieu et al., Kluwer Academic, Dordrecht (1999) 267-275, hepth/9512077.

[10] B. Craps and F. Roose, Anomalous D-brane and orientifold couplings from the boundary state, Phys. Lett. B445 (1998) 150-159, hep-th/9808074; (Non-) anomalous D-brane and O-plane couplings: the normal bundle, Phys. Lett. B450 (1999) 358-362, hep-th/9812149.

[11] B. Stefanski, Gravitational couplings for D-branes and O-planes, Nucl. Phys. B548 (1999) 275-290, hep-th/9812088.

[12] M. Bershadsky, V. Sadov and C. Vafa, D-branes and topological field theories, Nucl. Phys. B462 (1996) 420-434, hep-th/9511222.

[13] K. Dasgupta, D.P. Jatkar and S. Mukhi, Gravitational couplings and $Z_{2}$ orientifolds, Nucl. Phys. B523 (1998) 465-484, hep-th/9707224.

[14] K. Dasgupta and S. Mukhi, Anomaly inflow on orientifold planes, JHEP 03 (1998) 004, hep-th/9709219.

[15] A. Sagnotti, in Non-perturbative quantum field theory, ed. G. Mack et al., Pergamon, Oxford (1988) 521.

[16] G. Pradisi and A. Sagnotti, Open strings orbifolds, Phys. Lett. B216 (1989) 59-67.

[17] P. Horava, Strings on world sheet orbifolds, Nucl. Phys. B327 (1989) 461; Background duality of open string models, Phys. Lett. B231 (1989) 251.

[18] J. Dai, R. Leigh and J. Polchinski, New connections between string theories, Mod. Phys. Lett. A4 (1989) 2073-2083.

[19] M. Bianchi and A. Sagnotti, On the systematics of open string theories, Phys. Lett. B247 (1990) 517-524; Twist symmetry and open-string Wilson lines, Nucl. Phys. B361 (1991) 519-538.

[20] E.G. Gimon and J. Polchinski, Consistency conditions for orientifolds and Dmanifolds, Phys. Rev. D54 (1996) 1667-1676, hep-th/9601038.

[21] E.G. Gimon and C.V. Johnson, K3 orientifolds, Nucl. Phys. B477 (1996) 715745, hep-th/9604129.

[22] A. Dabholkar and J. Park, An orientifold of type IIB theory on K3, Nucl. Phys. B472 (1996) 207-220, hep-th/9602030; Strings on orientifolds, Nucl. Phys. B477 (1996) 701-714, hep-th/9604178.

[23] J. Polchinski, Tensors from K3 orientifolds, Phys. Rev. D55 (1997) 6423-6428, hep-th/9606165.

[24] L. Alvarez-Gaumé and E. Witten, Gravitational anomalies, Nucl. Phys. B234 (1984) 269-330. 
[25] A. Sagnotti, A note on the Green-Schwarz mechanism in open-string theories, Phys. Lett. B294 (1992) 196-203, hep-th/9210127.

[26] M. Berkooz, R. G. Leigh, J. Polchinski, J. H. Schwarz, N. Seiberg and E. Witten, Anomalies, dualities, and topology of $D=6 N=1$ superstring vacua, Nucl. Phys. B475 (1996) 115-148, hep-th/9605184.

[27] L.E. Ibáñez, R. Rabadan and A.M. Uranga, Anomalous U(1)'s in type I and type IIB $D=4$ N=1 string vacua, Nucl. Phys. B542 (1999) 112-138, hep-th/9808139.

[28] M.J. Duff, R. Minasian and E. Witten, Evidence for heterotic/heterotic duality, Nucl. Phys. B465 (1996) 413-438, hep-th/9601036.

[29] N. Seiberg and E. Witten, Comments on string dynamics in six dimensions, Nucl. Phys. B471 (1996) 121-134, hep-th/9603003.

[30] M.J. Duff, H. Lü and C.N. Pope, Heterotic phase transitions and singularities of the gauge dyonic string, Phys. Lett. B378 (1996) 101-106, hep-th/9603037.

[31] I. Antoniadis, C. Bachas and E. Dudas, Gauge couplings in four-dimensional type I string orbifolds, hep-th/9906039.

[32] J. Polchinski and Y. Cai, Consistency of open superstring theories, Nucl. Phys. B296 (1988) 91-128;

[33] D. Friedan, E. Martinec and S. Shenker, Conformal invariance, supersymmetry and string theory, Nucl. Phys. B271 (1986) 93-165.

[34] T. Inami, H. Kanno and T. Kubota, Hexagon gauge anomaly of the type-I superstring in the path integral method, Phys. Lett. B199 (1987) 389-394; Hexagon gauge anomaly and supermoduli in the path integral method of superstrings, Nucl. Phys. B308 (1988) 203-220.

[35] J. Liu, Gauge and gravitational anomaly cancellation in type-I superstring theory, Nucl. Phys. B362 (1991) 141-172.

[36] M.B. Green, J.H. Schwarz and E. Witten, Superstring theory: volume 2, Cambridge University Press (1987).

[37] E. Witten, Constraints on supersymmetry breaking, Nucl. Phys. B202 (1982) 253-316.

[38] M. Bertolini, R. Iengo and C.A. Scrucca, Electric and magnetic interaction of dyonic D-branes and odd spin structure, Nucl. Phys. B522 (1998) 193-213, hepth/9801110.

[39] J.H. Schwarz and A. Sen, Duality symmetric actions, Nucl. Phys. B411 (1994) 35-63, hep-th/9304154.

[40] P. Pasti, D. Sorokin and M. Tonin, Duality symmetric actions with manifest space-time symmetries, Phys. Rev. D52 (1995) 4277-4281, hep-th/9506109; On Lorentz invariant actions for chiral p-forms, Phys. Rev. D55 (1997) 6292-6298, hep-th/9611100.

[41] M.R. Douglas and G. Moore, D-branes, quivers and ALE instantons, hepth/9603167. 
[42] S. Ferrara, F. Riccioni and A. Sagnotti, Tensor and vector multiplets in sixdimensional supergravity, Nucl. Phys. B519 (1998) 115-140, hep-th/9711059.

[43] H. Nishino and E. Sezgin, New couplings of six-dimensional supergravity, Nucl. Phys. B505 (1997) 497-516, hep-th/9703075.

[44] F. Riccioni and A. Sagnotti, Self dual tensors in six-dimensional supergravity, hep-th/9812042.

[45] C. Angelantonj, M. Bianchi, G. Pradisi, A. Sagnotti and Ya.S. Stanev, Comments on Gepner models and type I vacua in string theory, Phys. Lett. B387 (1996) 743-749, hep-th/9607229.

[46] E. Witten, Five-branes and M-theory on an orbifold, Nucl. Phys. B463 (1996) 383-397, hep-th/9512219.

[47] G. Aldazabal, A. Font, L.E. Ibáñez, A.M. Uranga and G. Violero, Nonperturbative heterotic $D=6,4, N=1$ orbifold vacua, Nucl. Phys. B519 (1998) 239281, hep-th/9706158.

[48] P. Horava and E. Witten, Heterotic and Type I string dynamics from eleven dimensions, Nucl. Phys. B460 (1996) 506-524, hep-th/9510209. 